AperTO - Archivio Istituzionale Open Access dell'Università di Torino

Second-order ordinary differential equations with indefinite weight: the Neumann boundary value problem

This is a pre print version of the following article:

Original Citation:

Availability:

This version is available http://hdl.handle.net/2318/1509329

since 2016-06-24T12:44:53Z

Published version:

DOI:10.1007/s10231-013-0384-0

Terms of use:

Open Access

Anyone can freely access the full text of works made available as "Open Access". Works made available under a Creative Commons license can be used according to the terms and conditions of said license. Use of all other works requires consent of the right holder (author or publisher) if not exempted from copyright protection by the applicable law. 


\section{IIIS AperTO}

This is the author's final version of the contribution published as:

A. Boscaggin and F. Zanolin, Second order ordinary differential equations with indefinite weight: the Neumann boundary value problem, Ann. Mat. Pura Appl. (4) 194 (2015), 451-478, DOI 10.1007/s10231-013-0384-0

The publisher's version is available at:

http://link.springer.com/article/10.1007\%2Fs10231-013-0384-0

When citing, please refer to the published version.

Link to this full text:

http://link.springer.com/article/10.1007\%2Fs10231-013-0384-0 


\title{
Second order ordinary differential equations with indefinite weight: the Neumann boundary value problem
}

\author{
Alberto Boscaggin* \\ Department of Mathematics, University of Torino, \\ Via Carlo Alberto, 10, 10123 Torino - Italy \\ e-mail: alberto.boscaggin@unito.it \\ Fabio Zanolin ${ }^{\dagger}$ \\ Department of Mathematics and Computer Science, University of Udine, \\ via delle Scienze 206, 33100 Udine - Italy \\ e-mail: fabio.zanolin@uniud.it
}

\begin{abstract}
We study the second order nonlinear differential equation $u^{\prime \prime}+a(t) g(u)=0$, where $g$ is a continuously differentiable function of constant sign defined on an open interval $I \subseteq \mathbb{R}$ and $a(t)$ is a sign-changing weight function. We look for solutions $u(t)$ of the differential equation such that $u(t) \in I$, satisfying the Neumann boundary conditions. Special examples, considered in our model, are the equations with singularity, for $I=\mathbb{R}_{0}^{+}$and $g(u) \sim-u^{-\sigma}$, as well as the case of exponential nonlinearities, for $I=\mathbb{R}$ and $g(u) \sim \exp (u)$. The proofs are obtained by passing to an equivalent equation of the form $x^{\prime \prime}=f(x)\left(x^{\prime}\right)^{2}+a(t)$.
\end{abstract}

Mathematics Subject Classification. 34B15, 34B09.

Key words. Boundary value problems, indefinite weight, necessary and sufficient solvability conditions.

*The author acknowledges the support of the project "Equazioni Differenziali Nonlineari e Applicazioni”, Department of Mathematics, University of Torino.

${ }^{\dagger}$ The author acknowledges the support of the PRIN-2009 project "Ordinary Differential Equations and Applications". 


\section{Introduction}

This article is devoted to the study of boundary value problems associated to the second order nonlinear differential equation

$$
u^{\prime \prime}+a(t) g(u)=0
$$

Throughout the paper we assume that $g: I \rightarrow \mathbb{R}$ is a continuously differentiable function defined on an open interval $I \subseteq \mathbb{R}$ and such that $g(s) \neq 0$, for every $s \in I$. The coefficient (weight function) $a(t)$ is supposed to be a Lebesgue integrable function (not identically zero!) defined on a compact interval $[0, T]$ and solutions are meant in the Carathéodory sense [23].

Equation (1.1), in spite of its simple looking structure, covers a broad number of different situations. A typical example that will be discussed below is when $\left.\left.I=\mathbb{R}_{0}^{+}:=\right] 0,+\infty\right)$. In this case, a natural choice for $g$ is given by

$$
g(s)=s^{\alpha}, \quad \alpha \neq 0 .
$$

When $\alpha>0$, although the nonlinearity can be continuously extended to the whole real line, the study of solutions of (1.1) with range in $I$ leads to the search of positive solutions, a topic which has been widely investigated for its relevance in the applications. On the other hand, when $\alpha<0$, we are led to study an equation with a singularity at the origin. Less standard examples, which will be also discussed later, are for $I=\mathbb{R}$. In this setting, one can study, for instance, an equation like $u^{\prime \prime}+a(t) \exp (u)=0$.

Our main interest will be the search of solutions $u:[0, T] \rightarrow I$ of (1.1) satisfying the Neumann boundary conditions

$$
u^{\prime}(0)=u^{\prime}(T)=0
$$

The case of periodic boundary conditions could be considered, as well. It is a known fact that there are strong similarities and connections between the periodic and the Neumann boundary value problems for equation (1.1) (besides the obvious fact that, in both cases, $\lambda_{0}=0$ is the principal eigenvalue for the operator $-u^{\prime \prime}$, with the corresponding eigenspace made up by the constant functions). For instance, if we extend $a(t)$ to the whole real line as a $2 T$-periodic even function, then any solution of (1.1)-(1.2) can be extended to an even $2 T$-periodic solution of the same equation. Moreover, as we shall see below, usual necessary and/or sufficient conditions on $a(t)$ for the existence of solutions to the Neumann or to the periodic problem turn out to be the same.

A first and very elementary observation is that any solutions of (1.1) satisfying the Neumann (as well as the periodic) boundary conditions is such that $\int_{0}^{T} u^{\prime \prime}(t) d t=$ 
0 and, therefore,

$$
\int_{0}^{T} a(t) g(u(t)) d t=0
$$

Since $g(s)$ is of constant sign on $I$, we immediately obtain a first necessary condition on the weight function, namely

$$
a^{+}(t) \not \equiv 0 \quad \text { and } \quad a^{-}(t) \not \equiv 0,
$$

that is, $a(t)$ is sign-indefinite (see the end of this Introduction for some clarifications). A second necessary condition can be obtained under the additional assumption that $g^{\prime}(s)$ never vanishes on the interval $I$. Indeed, writing

$$
-\frac{u^{\prime \prime}(t)}{g(u(t))}=a(t)
$$

integrating by parts on $[0, T]$ and using the boundary conditions, we get

$$
\int_{0}^{T} a(t) d t=-\left[\frac{u^{\prime}(t)}{g(u(t))}\right]_{0}^{T}-\int_{0}^{T} \frac{u^{\prime}(t)^{2}}{g(u(t))^{2}} g^{\prime}(u(t)) d t=-\int_{0}^{T} \frac{u^{\prime}(t)^{2}}{g(u(t))^{2}} g^{\prime}(u(t)) d t .
$$

Since $u(t)$ cannot be constant (otherwise $a(t) \equiv 0$ ), we obtain that

$$
\int_{0}^{T} a(t) d t \neq 0 \quad \text { and } \quad \operatorname{sgn}\left(\int_{0}^{T} a(t) d t\right)=-\left(\left.\operatorname{sgn} g^{\prime}\right|_{I}\right) .
$$

Notice that, if we assume that $g^{\prime}(s)$ has constant sign on $I$, then it is not restrictive to suppose that

$$
g^{\prime}(s)>0, \quad \forall s \in I .
$$

In this case, the necessary condition (1.3) becomes

$$
\int_{0}^{T} a(t) d t<0
$$

In the past fifty years a great deal of research has been devoted to the study of nonlinear equations with a sign-indefinite weight (see, for instance, [9, Introduction] for a brief survey on this subject). The investigation of the case in which the nonlinear term $g(s)$ has superlinear growth at infinity (namely, $g(s) \sim|s|^{p-1} s$, with $p>1$ ) led to multiplicity results of oscillatory solutions for various boundary value problems associated to $(1.1)$ (see $[13,30,34]$ and the references therein). The search of positive solutions has been addressed both to the case of ODEs and to nonlinear elliptic PDEs of the form

$$
\Delta u-\lambda u+a(x) g(u)=0, \quad x \in \Omega \subset \mathbb{R}^{N},
$$


under different conditions for $\left.u\right|_{\partial \Omega}$ (see $[1,2,3,4,5,6]$ for some classical results in this direction). In particular, multiplicity results for (1.4), with Dirichlet boundary conditions on a bounded domain $\Omega$, have been obtained in $[7,17,19,21,28]$ in the superlinear case $g(s) \sim s^{p}$, with $p>1$. For (1.4) with $\lambda=0$, a large number of positive solutions for the Dirichlet problem have been found in [7, 17, 19, 20] when $a(x)$ has a certain number of large negative humps. However, concerning the Neumann/periodic problem for (1.1) or the Neumann problem for

$$
\Delta u+a(x) g(u)=0, \quad x \in \Omega \subset \mathbb{R}^{N},
$$

less results seem to be available (see, for instance [3, 4, 6, 8, 9, 10, 32]). In [4], Bandle, Pozio and Tesei studied the Neumann problem for equation (1.5) for a nonlinearity $g(s)$ which behaves like $s^{p}$ with $0<p<1$ and proved that the condition

$$
a^{+}(x) \not \equiv 0, \quad a^{-}(x) \not \equiv 0 \quad \text { and } \quad \int_{\Omega} a(x) d x<0
$$

is necessary and sufficient for the existence of a positive solution. A uniqueness result was also provided for $g(s)$ concave. In [6], Berestycki, Capuzzo-Dolcetta and Nirenberg considered equation (1.5) for $g(s)=s^{p}$ with $p>1$ and proved that (1.6) is again necessary and sufficient for the existence of a positive solution to the Neumann problem.

As observed before, the search of positive solutions, although quite relevant from the point of view of the applications, does not represent the only possible situation in our setting. The case $I=\mathbb{R}_{0}^{+}$allows to treat differential equations with singularities at the origin, like in $[12,16]$. In [12], Bravo and Torres studied the equation with singularity

$$
u^{\prime \prime}-\frac{a(t)}{u^{3}}=0,
$$

for $a(t)$ a $T$-periodic piecewise constant function of the form $a(t)=a_{+}>0$ for $0 \leq t<\tau$ and $a(t)=-a_{-}<0$ for $\tau \leq t<T$. Again, condition $\left(A_{1}\right)$ was shown to be necessary and sufficient for the existence of a (positive) periodic solution (and a uniqueness result was also obtained). A recent extension of this existence result, to the equation $u^{\prime \prime}-\frac{a(t)}{u^{\sigma}}=0$ for the case of a piecewise constant periodic weight function with an arbitrary number of humps (and negative mean value) and $\sigma>1$, has been achieved by Ureña [35].

As a final example we consider the case in which $I=\mathbb{R}$ and $g(s)=\exp (s)$. In [27], Le and Schmitt proved the existence of solutions for the Neumann problem associated to (1.5) as well as for the periodic problem associated to $u^{\prime \prime}+a(t) \exp (u)=$ 0 . Again condition (1.6) (respectively $\left.\left(A_{0}\right)-\left(A_{1}\right)\right)$ turns out to be necessary and sufficient for the solvability of the problem. 
The above discussion suggests a couple of natural questions toward which address a possible research, namely:

- whether it is possible to provide a unified setting for the study of the different situations just described, namely, the case of positive solutions, equations with singularities, nonlinearities of constant sign defined on the whole real line;

- to what extent an average condition of the form $\left(A_{1}\right)$ is sufficient for the existence of solutions, possibly also in cases in which $g(s)$ is not monotone (and hence $\left(A_{1}\right)$ is not necessary).

The main purpose of the present paper is to provide partial answers to both the questions. Although we believe that some results can be extended to the periodic setting, we prefer to focus our attention to the Neumann problem for (1.1), which looks easier to deal with. Moreover, for simplicity, we shall restrict ourselves to the case in which the weight function $a(t)$ has only one positive hump and one negative hump. In this framework, by changing, if necessary, $t$ with $T-t$ we can always enter in the setting of the following condition $\left(A_{2}\right)$ :

$\left(A_{2}\right) \quad a(t) \geq 0$, for a.e. $t \in[0, \tau]$ and $a(t) \leq 0$, for a.e. $t \in[\tau, T]$,

for some $\tau \in] 0, T[$. Such an assumption on the weight function is, in some sense, a natural one within the approach that we follow to prove our existence results. More details about the argument of our proof will be provided at the end of Section 3. We point out, however, that even if the shape of $a(t)$ is rather simple, on the other hand we do not require any further special condition (like the simplicity of its zeros). In particular $a(t)$ may vanish on subsets of $[0, T]$ with positive measure, like subintervals.

The paper is organized as follows. In Section 2 we introduce a second order auxiliary equation of the form $x^{\prime \prime}=f(x)\left(x^{\prime}\right)^{2}+a(t)$ which allows us to treat in a unified setting different cases of equation (1.1) (including the problems described above). Such an approach seems new for this kind of problems. Analogous considerations have been recently developed in a different direction, by transforming autonomous equations like $x^{\prime \prime}+f(x)\left(x^{\prime}\right)^{2}+\phi(x)=0$ to second order conservative equations $u^{\prime \prime}+\psi(u)=0$. In Section 3 we state our main theorems (Theorem 3.1 and related results) and show some immediate applications. Section 4 is devoted to the proof of some technical lemmas and estimates from which the proof of Theorem 3.1 follows. Finally, in Section 5 we give some final remarks.

Throughout the paper, the following notation is used. For the weight function $a(t)$, we define $a^{ \pm}: I \rightarrow \mathbb{R}$ as

$$
a^{+}(t):=\max \{a(t), 0\}, \quad a^{-}(t):=\max \{-a(t)(x), 0\},
$$


so that

$$
a(t)=a^{+}(t)-a^{-}(t), \quad|a(t)|=a^{+}(t)-a^{-}(t), \quad \forall t \in I .
$$

Since $a \in L^{1}(0, T)$, equalities/inequalities are understood up to sets of zero measure. For instance, $a \equiv 0$ on an interval $\left[t_{0}, t_{1}\right]$ means that $a(t)=0$ for a.e. $t \in\left[t_{0}, t_{1}\right]$, namely $\int_{t_{0}}^{t_{1}}|a(t)| d t=0$. Accordingly, $a \neq 0$ on $\left[t_{0}, t_{1}\right]$ if and only if $\int_{t_{0}}^{t_{1}}|a(t)| d t \neq 0$. Finally, the symbols $|\cdot|_{1}$ and $|\cdot|_{\infty}$ stand, respectively, for the $L^{1}$-norm and the $L^{\infty}$-norm (the domain being clear from the context or explicitly indicated).

\section{An equivalent formulation of the problem}

Let $I \subseteq \mathbb{R}$ be an open interval and $g: I \rightarrow \mathbb{R}$ be a continuously differentiable function such that $g(s) \neq 0$, for every $s \in I$. We deal with the second order nonlinear ODE

$$
u^{\prime \prime}+a(t) g(u)=0
$$

where $a \in L^{1}(0, T)$. Associated to (2.1) the Neumann problem

$$
u^{\prime}(0)=u^{\prime}(T)=0
$$

is considered. By a solution of (2.1) we mean a function $u: \operatorname{dom}(u) \rightarrow I$ satisfying (2.1) in the generalized (Carathéodory) sense.

Setting

$$
h(s):=-\frac{1}{g(s)},
$$

equation (2.1) is obviously equivalent to

$$
u^{\prime \prime}=\frac{a(t)}{h(u)} .
$$

Let $H: I \rightarrow J:=H(I)$ be a primitive of $h$. Observe that $H$ is a diffeomorphism of the interval $I$ onto the open interval $J$. Then, by simple manipulations one can check that equation (2.3) is equivalent to the forced equation

$$
x^{\prime \prime}=f(x)\left(x^{\prime}\right)^{2}+a(t)
$$

where $f: J \rightarrow \mathbb{R}$ is defined as

$$
f(x):=g^{\prime}\left(H^{-1}(x)\right), \quad \forall x \in J
$$

and

$$
x(t):=H(u(t))
$$


Moreover, it is straightforward to see that $u:[0, T] \rightarrow I$ is a solution of (2.1)-(2.2) if and only if $x:[0, T] \rightarrow J$ is a solution of (2.4) satisfying the Neumann boundary conditions

$$
x^{\prime}(0)=x^{\prime}(T)=0
$$

(a similar equivalence holds true if one looks for $T$-periodic solutions).

Equation (2.4) appears as a perturbation (by means of the forcing therm $a(t)$ ) of the conservative equation

$$
x^{\prime \prime}-f(x)\left(x^{\prime}\right)^{2}=0
$$

which is considered in some classical textbooks like [25, Vol. 1, Ex. 6.51, p. 554]. It is an obvious fact that all the constant functions $x(t) \equiv c \in J$ are solutions of (2.6). For this reason, in order to avoid trivialities, when dealing with problem (2.4)-(2.5) we will always suppose that $a(t) \not \equiv 0$.

Notice that from equation (2.4) one can easily recover the average condition on $a(t)$ in order to have solutions satisfying the Neumann (or the periodic) conditions. Indeed, $g^{\prime}(s)$ never vanishes on the interval $I$ if and only if $f(x)$ has constant sign on $J$. Therefore, just integrating both sides of (2.4) we get again (1.3) (that is, in the case $\left.f>0,\left(A_{1}\right)\right)$. More formally, this is expressed by the following result which refers directly to equation (2.4).

Lemma 2.1 Let $J \subseteq \mathbb{R}$ be an open interval and let $f: J \rightarrow \mathbb{R}$ be a continuous function such that $f(x) \neq 0$, for all $x \in J$. Let $a(t) \not \equiv 0$. Then (2.4)-(2.5) has a solution only if

$$
\int_{0}^{T} a(t) d t \neq 0 \quad \text { and } \quad \operatorname{sgn}\left(\int_{0}^{T} a(t) d t\right)=-\left(\left.\operatorname{sgn} f\right|_{J}\right) .
$$

Proof. Let $x:[0, T] \rightarrow J$ be a solution of (2.4)-(2.5). Integrating the equation on $[0, T]$ we obtain $-\int_{0}^{T} f(x(t)) x^{\prime}(t)^{2} d t=\int_{0}^{T} a(t) d t$, hence $\int_{0}^{T} a(t) d t \leq 0$ or $\int_{0}^{T} a(t) d t \geq 0$ according to the fact that $f>0$ or $f<0$ on $J$. On the other hand, $\int_{0}^{T} a(t) d t \neq 0$. In fact, on the contrary, we would have that $x^{\prime}(t) \equiv 0$, that is $x(t)$ is a constant solution, but this is prevented by the fact that $a(t) \not \equiv 0$. Hence the thesis follows.

The reason why the condition $\left(A_{0}\right)$ is necessary also for the solvability of problem (2.4)-(2.5) is perhaps less evident. However, it follows by the following result.

Lemma 2.2 Let $J \subseteq \mathbb{R}$ be an open interval and let $f: J \rightarrow \mathbb{R}$ be a continuous function. Let $a(t) \not \equiv 0$. Then (2.4)-(2.5) has a solution only if $\left(A_{0}\right)$ holds. 
The proof of Lemma 2.2 is an immediate consequence of the next two results which will be also applied in Section 4. In Lemma 2.3 and Lemma 2.4 below we do not need to assume that the solutions of the initial problems associated to (2.4) are globally defined the whole interval where $a(t)$ is defined.

Lemma 2.3 Let $f: J(\subseteq \mathbb{R}) \rightarrow \mathbb{R}$ be a continuous function and let $a(\cdot) \in L^{1}\left(t_{0}, t_{1}\right)$ be such that $a(t) \geq 0$ for a.e. $t \in\left[t_{0}, t_{1}\right]$. Suppose that $x(t)$ is a solution of (2.4) such that $x^{\prime}\left(t_{0}\right)=0$. Let $\left.\left.s \in\right] t_{0}, t_{1}\right]$ be such that $x(s)$ is defined and $\int_{t_{0}}^{s} a(\xi) d \xi>0$. Then $x^{\prime}(t)>0$ for all $t \in\left[s, t_{1}\right]$ where $x(\cdot)$ is defined.

Lemma 2.4 Let $f: J(\subseteq \mathbb{R}) \rightarrow \mathbb{R}$ be a continuous function and let $a(\cdot) \in L^{1}\left(t_{0}, t_{1}\right)$ be such that $a(t) \leq 0$ for a.e. $t \in\left[t_{0}, t_{1}\right]$. Suppose that $x(t)$ is a solution of $(2.4)$ such that $x^{\prime}\left(t_{0}\right)=0$. Let $\left.\left.s \in\right] t_{0}, t_{1}\right]$ be such that $x(s)$ is defined and $\int_{t_{0}}^{s} a(\xi) d \xi<0$. Then $x^{\prime}(t)<0$ for all $t \in\left[s, t_{1}\right]$ where $x(\cdot)$ is defined.

Proof. Due to the symmetry of the two statements, we prove only Lemma 2.3. We define $A_{t_{0}}(t):=\int_{t_{0}}^{t} a(\xi) d \xi$ and $\sigma_{0}:=\max \left\{t \geq t_{0}: A_{t_{0}}(t)=0\right\}$. If there exists $\left.s \in] t_{0}, t_{1}\right]$ such that $A_{t_{0}}(s)>0$, then $\sigma_{0}<t_{1}$ and $A_{t_{0}}(t)>0$ for all $\left.\left.t \in\right] \sigma_{0}, t_{1}\right]$. Hence, proving Lemma 2.3 is the same like proving that $x^{\prime}(t)>0$ for every $t>\sigma_{0}$ (where $x(\cdot)$ is defined).

First of all, we observe that $x^{\prime}(t) \geq 0, \forall t \in\left[t_{0}, t_{1}\right] \cap \operatorname{dom}(x)$. Indeed, if, by contradiction, $x^{\prime}(t)<0$ for some $\left.t \in\right] t_{0}, t_{1}[$, then we can find a maximal interval ]$s_{0}, s_{1}[\subseteq] t_{0}, t_{1}\left[\right.$ such that $x^{\prime}(t)<0$ for all $\left.t \in\right] s_{0}, s_{1}\left[\right.$ and with $x^{\prime}\left(s_{0}\right)=0$. We take now $t, t^{*}$ with $s_{0}<t<t^{*}<s_{1}$ and integrate

$$
\frac{x^{\prime \prime}(s)}{x^{\prime}(s)}=f(x(s)) x^{\prime}(s)+\frac{a(s)}{x^{\prime}(s)} \leq f(x(s)) x^{\prime}(s)
$$

on $\left[t, t^{*}\right]$, so that we get

$$
\log \left|x^{\prime}\left(t^{*}\right)\right|-\log \left|x^{\prime}(t)\right| \leq \int_{x(t)}^{x\left(t^{*}\right)} f(\xi) d \xi=-F_{r}(x(t)),
$$

where we have set $F_{r}(x):=\int_{r}^{x} f(\xi) d \xi$, for $r:=x\left(t^{*}\right)$. Then we have

$$
\left.\left.-\log \left|x^{\prime}(t)\right| \leq K:=-\log \left|x^{\prime}\left(t^{*}\right)\right|+\max _{x \in J^{\prime}}\left|F_{r}(x)\right|, \quad \forall t \in\right] s_{0}, t^{*}\right],
$$

where $J^{\prime}$ is the compact interval $J^{\prime}:=\left\{x(\xi): \xi \in\left[s_{0}, t^{*}\right]\right\} \subseteq J$. From the last inequality, a contradiction is immediately achieved, by taking the limit as $t \rightarrow s_{0}^{+}$. Now that we have proved that $x^{\prime}(t) \geq 0, \forall t \in\left[t_{0}, t_{1}\right] \cap \operatorname{dom}(x)$, we can proceed further. 
Suppose, by contradiction that there is $\left.\left.s_{1} \in\right] \sigma_{0}, t_{1}\right]$ such that $x(\cdot)$ is defined on $\left[t_{0}, s_{1}\right]$ and $x^{\prime}\left(s_{1}\right)=0$. We integrate (2.4) on $\left[s_{1}-t, s_{1}\right]$ and obtain

$$
-x^{\prime}\left(s_{1}-t\right)=\int_{s_{1}-t}^{s_{1}} f(x(\xi))\left(x^{\prime}(\xi)\right)^{2} d \xi+\int_{s_{1}-t}^{s_{1}} a(\xi) d \xi
$$

that is, for $v(t):=x^{\prime}\left(s_{1}-t\right)$,

$$
0 \leq v(t)=\int_{0}^{t}-f\left(x\left(s_{1}-\theta\right)\right) v(\theta)^{2} d \theta-\int_{s_{1}-t}^{s_{1}} a(\xi) d \xi \leq M \int_{0}^{t} v(\theta) d \theta,
$$

for $M>0$ a suitable constant which bounds $\left|f(x(t)) x^{\prime}(t)\right|$ on $\left[t_{0}, s_{1}\right]$. Gronwall's inequality implies that $v(t) \equiv 0$ on $\left[t_{0}, s_{1}\right]$, that is $x(t)$ is constant. This is a contradiction to the fact that $a \not \equiv 0$ on $\left[t_{0}, s_{1}\right]$.

A more straightforward manner to prove Lemma 2.2 can be provided too, once we observe that equation (2.4) is equivalent to

$$
\frac{d}{d t}\left(x^{\prime} \exp (-F(x))\right)=a(t) \exp (-F(x))
$$

where $F: J \rightarrow \mathbb{R}$ is a primitive of $f(x)$ on $J$. In this situation, it is clear that any solution of (2.8) satisfying the Neumann boundary condition (as well as any $T$-periodic solution) is such that $\int_{0}^{T} a(t) \exp (-F(x(t))) d t=0$. Hence $\left(A_{0}\right)$ must be satisfied.

The equivalence between (2.4) and (2.8) allows us to provide an immediate proof of the fact that any Cauchy problem associated to (2.4) has a unique (noncontinuable) solution. Indeed, it is sufficient to observe that (2.8) is equivalent to the first order planar system

$$
\left\{\begin{array}{l}
x^{\prime}=y \exp (F(x)) \\
y^{\prime}=a(t) \exp (-F(x))
\end{array}\right.
$$

which is of the form $z^{\prime}=X(t, z)$, with $X(t, \cdot)$ defined on the open domain $J \times$ $\mathbb{R} \subseteq \mathbb{R}^{2}$, locally Lipschitz continuous in $z=(x, y)$ and satisfying the Carathéodory conditions [23].

As a final remark, we also notice that via equation (2.8) one can cast (2.4) back into the form of (2.1). In fact, if we denote by $\mathscr{F}(x)$ a primitive of $\exp (-F(x))$ on the (open) interval $J$, we see that $\mathscr{F}$ is a strictly increasing diffeomorphism of $J$ onto an open interval $I^{\prime}:=\mathscr{F}(J)$. Then $x(t)$ is a solution to (2.4) if and only if $w(t):=\mathscr{F}(x(t))$ is a solution of

$$
w^{\prime \prime}+a(t) \mathrm{g}(w)=0,
$$


where $\mathrm{g}: I^{\prime} \rightarrow \mathbb{R}$ is a continuously differentiable function of constant sign, defined as

$$
\mathrm{g}(s):=-\exp \left(-F\left(\mathscr{F}^{-1}(s)\right)\right)
$$

Remark 2.1 The transformation from (2.4) to (2.9) works for more general equations too. For instance, if we start from equation

$$
x^{\prime \prime}=f(x)\left(x^{\prime}\right)^{2}+\phi(t, x)
$$

then the same change of variables leads to

$$
w^{\prime \prime}+\psi(t, w)=0
$$

with $\psi(t, s):=-\phi\left(t, \mathscr{F}^{-1}(s)\right) \exp \left(-F\left(\mathscr{F}^{-1}(s)\right)\right)$. Analogous considerations have been developed in order to transform the second order autonomous equation

$$
x^{\prime \prime}+f(x)\left(x^{\prime}\right)^{2}+\phi(x)=0
$$

to an equation of the form $w^{\prime \prime}+\psi(w)=0$ (see [33]). Besides the non autonomous nature of (2.4) (or (2.10)), compared to (2.12), the main feature of our research is that for the equivalent equation (2.9) (or (2.11)), the nonlinear term has constant sign in $w$ (and the sign change is in the $t$-variable), whence in [33], as well as in related papers studying the period map of planar centers, the nonlinear terms $\phi$ and $\psi$ change their sign. In this connection we also recall a classical work of Chicone [14], where the Neumann problem for a class of equations included in (2.12) is considered.

At this point we reconsider the most classical examples of equation (2.1) discussed in the Introduction and we look for their equivalent formulation in the version of (2.4). Namely, we obtain:

(I1) Let $g(s)=s^{p}$, with $0<p<1$, for $I=\mathbb{R}_{0}^{+}$. Then $f(x)=\frac{p}{(p-1) x}$, for $J=\mathbb{R}_{0}^{-}:=\left(-\infty, 0\left[\right.\right.$. The function $H: I \rightarrow J$, with $H(s)=-\frac{1}{1-p} s^{1-p}$, is strictly decreasing with $H\left(0^{+}\right)=0^{-}$and $H(+\infty)=-\infty$;

(I2) Let $g(s)=s^{p}$, with $p>1$, for $I=\mathbb{R}_{0}^{+}$. Then $f(x)=\frac{p}{(p-1) x}$, for $J=\mathbb{R}_{0}^{+}$. The function $H: I \rightarrow J$, with $H(s)=\frac{1}{(p-1) s^{p-1}}$, is strictly decreasing with $H\left(0^{+}\right)=+\infty$ and $H(+\infty)=0^{+}$; 
(I3) Let $g(s)=-s^{-\sigma}$, with $\sigma>0$, for $I=\mathbb{R}_{0}^{+}$. Then $f(x)=\frac{\sigma}{(\sigma+1) x}$, for $J=\mathbb{R}_{0}^{+}$. The function $H: I \rightarrow J$, with $H(s)=\frac{1}{(\sigma+1)} s^{\sigma+1}$, is strictly increasing with $H\left(0^{+}\right)=0^{+}$and $H(+\infty)=+\infty$;

(I4) Let $g(s)=\exp s$, for $I=\mathbb{R}$. Then $f(x)=\frac{1}{x}$, for $J=\mathbb{R}_{0}^{+}$. The function $H: I \rightarrow J$, with $H(s)=\exp (-s)$ is strictly decreasing with $H(-\infty)=+\infty$ and $H(+\infty)=0^{+}$.

It is easy to show that coming back from the form (2.4) to the equation (2.9) we obtain a similar structure as the initial one. For instance, from the equation $u^{\prime \prime}+$ $a(t) \exp (u)=0$ of the form (2.1) we pass to the equivalent equation $x^{\prime \prime}=\frac{1}{x}\left(x^{\prime}\right)^{2}+$ $a(t)$ of the form (2.4) and then to the equation $w^{\prime \prime}-a(t) \exp (-w)=0$ of the form (2.9), which is precisely the starting equation with the substitution $w=-u$.

\section{The main results}

In this section we state the main results of the paper.

We first recall, for the reader's convenience, the assumptions on the weight function $a \in L^{1}(0, T)$, namely:

$$
a^{+}(t) \not \equiv 0 \quad \text { and } \quad a^{-}(t) \not \equiv 0,
$$

$$
\int_{0}^{T} a(t) d t<0
$$

$\left(A_{2}\right) \quad a(t) \geq 0$, for a.e. $t \in[0, \tau]$ and $a(t) \leq 0$, for a.e. $t \in[\tau, T]$,

for some $\tau \in] 0, T[$.

As for the conditions on $f$, they are suggested by the last three examples at the end of Section 3. Accordingly, henceforth we deal with $f: \mathbb{R}_{0}^{+} \rightarrow \mathbb{R}$ a continuous function and we also define

$$
F(x):=\int_{1}^{x} f(\xi) d \xi
$$

Concerning the behavior of $f$ at infinity, we introduce the following conditions:

$$
f(+\infty):=\lim _{x \rightarrow+\infty} f(x)=0,
$$

$$
\liminf _{x \rightarrow+\infty} F(x)>-\infty
$$


Clearly, $\left(F_{\infty}\right)$ is always satisfied when $f(x) \geq 0$ for $x$ large. Concerning the behavior of $f$ and $F$ at $x=0^{+}$, the situation is more involved (and also related to the existence of solutions presenting a blow-up). In the statements of our results, possible conditions are the following:

$$
\left.\left.f\left(0^{+}\right):=\lim _{x \rightarrow 0^{+}} f(x) \in\right] 0,+\infty\right],
$$

$$
\limsup _{x \rightarrow 0^{+}} F(x)<+\infty .
$$

Observe that $\left(F_{0}\right)$ is always satisfied when $f(x) \geq 0$ for $x$ small. Hence, in particular, $\left(f_{0}\right)$ implies $\left(F_{0}\right)$. A third hypothesis (see $(A F)$ below) relates the behavior of $F(x)$ as $x \rightarrow 0^{+}$with the infinitesimal order of

$$
A(t):=\int_{0}^{t} a(\xi) d \xi
$$

as $t \rightarrow 0^{+}$. In such a case, we also suppose that $A(t)>0$ for $\left.\left.t \in\right] 0, \tau\right]$; such an assumption is not restrictive (otherwise, if $a \equiv 0$ on $\left[0, \sigma_{0}\right]$ it will be sufficient to consider the Neumann boundary conditions on $\left[\sigma_{0}, T\right]$ and then extend the solution to $[0, T]$ by taking constant values on $\left.\left[0, \sigma_{0}\right]\right)$. Namely, we have:

there exists $\theta>0$ such that

$$
\lim _{t \rightarrow 0^{+}} \frac{\exp [F((1+\theta) t A(t))]}{A(t)}=0^{+} .
$$

Observe that $(A F)$ implies that $F\left(0^{+}\right)=-\infty$ and hence $\left(F_{0}\right)$.

We are now in position to state our main results for the Neumann problem

$$
\left\{\begin{array}{l}
x^{\prime \prime}=f(x)\left(x^{\prime}\right)^{2}+a(t) \\
x^{\prime}(0)=x^{\prime}(T)=0
\end{array}\right.
$$

Theorem 3.1 Let $a(\cdot) \in L^{1}(0, T)$ satisfy $\left(A_{0}\right),\left(A_{1}\right)$ and $\left(A_{2}\right)$. Let $f: \mathbb{R}_{0}^{+} \rightarrow \mathbb{R}$ be a continuous function satisfying $\left(f_{\infty}\right),\left(F_{\infty}\right),\left(f_{0}\right)$ and $(A F)$. Then problem (3.1) has at least one (increasing) solution.

Remark 3.1 The technical condition $(A F)$ is always satisfied (for any $\theta>0$ ) when

$$
\exists k \geq 1: F(x) \sim \log \left(x^{k}\right) \text { as } x \rightarrow 0^{+},
$$

that is, when (for some $k \geq 1$ ) $f(x) \sim k / x$ for $x \rightarrow 0^{+}$. Notice that in this situation we do not need additional assumptions on $A(t)$. This possibility, however, does not 
cover the case (I3) of equations with a singularity discussed at the end of Section 2 (since $k=\sigma /(\sigma+1)<1)$.

On the other hand, we can notice that $(A F)$ can be simplified when we have some information on the infinitesimal order of $A(t)$ as $t \rightarrow 0^{+}$. For instance, if we suppose that $a(t)$ is continuous and positive in a suitable interval $] 0, \delta[$ with $\delta<\tau$, and assume that

$$
\exists c>0, \alpha>0: A(t) \sim c t^{\alpha} \quad \text { as } t \rightarrow 0^{+}
$$

then $(A F)$ holds if and only if

$$
\lim _{x \rightarrow 0^{+}}\left(F(x)-\frac{\alpha}{\alpha+1} \log (x)\right)=-\infty
$$

(notice that the $\theta$-parameter as well as the constant $c$ are inessential, due to the properties of the logarithm).

A variant of Theorem 3.1 can be obtained if we have further information about the existence of solutions exhibiting blow-up. Namely, we have the following.

Theorem 3.2 Let $a(\cdot) \in L^{1}(0, T)$ satisfy $\left(A_{0}\right),\left(A_{1}\right)$ and $\left(A_{2}\right)$. Let $f: \mathbb{R}_{0}^{+} \rightarrow \mathbb{R}$ be a continuous function satisfying $\left(f_{\infty}\right),\left(F_{\infty}\right)$ and $\left(F_{0}\right)$. Suppose, moreover, that there exists $r>0$ such that the solution of the differential equation, satisfying the initial condition $x(0)=r, x^{\prime}(0)=0$, is not defined on $[0, \tau]$. Then problem (3.1) has at least one (increasing) solution.

We show now how the results obtained for (3.1) can be applied to the problem

$$
\left\{\begin{array}{l}
u^{\prime \prime}+a(t) g(u)=0 \\
u^{\prime}(0)=u^{\prime}(T)=0
\end{array}\right.
$$

To this aim, we consider three different examples which mimic the cases of positive solutions for superlinear equations (see the example (I2) at the end of Section 2), equations with a singularity (see (I3)) and exponential type nonlinearities (that is, (I4)). For all the next examples, we suppose that $a(t)$ satisfies $\left(A_{0}\right),\left(A_{1}\right)$ and $\left(A_{2}\right)$.

Example 3.1 Let $g: \mathbb{R}^{+} \rightarrow \mathbb{R}^{+}:=[0,+\infty)$ be a $C^{1}$-function with $g(0)=0$, $g(s)>0$ for $s>0$. If we suppose that

$$
\int_{0}^{1} \frac{1}{g}=+\infty, \quad \int_{1}^{+\infty} \frac{1}{g}<\infty,
$$

we can take a primitive $H$ of $-1 / g$ such that $H\left(\mathbb{R}_{0}^{+}\right)=\mathbb{R}_{0}^{+}$. Note that $H(s)$ is a strictly decreasing function with $H\left(0^{+}\right)=+\infty$ and $H(+\infty)=0^{+}$, that is a strictly 
decreasing diffeomorphism of $\mathbb{R}_{0}^{+}$onto itself. Accordingly, $\left(f_{\infty}\right)$ and $\left(f_{0}\right)$ hold if and only if

$$
\left.\left.g^{\prime}(0)=0 \quad \text { and } \quad g^{\prime}(+\infty) \in\right] 0,+\infty\right]
$$

As a next step, we consider the primitive $F(x)=\int_{1}^{x} f(\xi) d \xi$ for $f(x)=g^{\prime}\left(H^{-1}(x)\right)$. An easy computation yields

$$
\begin{aligned}
F(x) & =\int_{1}^{x} g^{\prime}\left(H^{-1}(\xi)\right) d \xi=\int_{c}^{H^{-1}(x)} g^{\prime}(u) h(u) d u=-\int_{c}^{H^{-1}(x)} \frac{g^{\prime}(u)}{g(u)} d u \\
& =K-\log \left(g\left(H^{-1}(x)\right)\right),
\end{aligned}
$$

for $c:=H^{-1}(1)$ and $K:=\log g(c)$. As a consequence,

$$
F(+\infty)=K-\log \left(g\left(0^{+}\right)\right)=+\infty,
$$

and thus $\left(F_{\infty}\right)$ holds. Moreover,

$$
F\left(0^{+}\right)=K-\log (g(+\infty)) .
$$

Hence $\left(F_{0}\right)$ is satisfied provided that $g(+\infty)>0$ (an assumption which holds with $g(+\infty)=+\infty$ when the second condition in (3.6) is satisfied).

We plan to apply Theorem 3.2. To this aim, we observe that a blow-up solution as required in Theorem 3.2 corresponds to a solution of $u^{\prime \prime}+a(t) g(u)=0$ satisfying the initial condition $u(0)=u_{0}>0$ and $u^{\prime}(0)=0$ and such that $u(t) \leq 0$ for some $t \leq \tau$. Solutions of this form always exist when $\liminf _{x \rightarrow+\infty} g(x) / x$ is large enough. This assertion can be checked in different ways. A first possibility is to use a Sturm comparison argument (with respect to the linear equation $u^{\prime \prime}+\lambda a(t) u=0$ ) as in the proof of [18, Lemma 4.3]. Alternatively, one can observe that [26, Corollary 3.6] guarantees the existence of a solution to $u^{\prime \prime}+a(t) g(u)=0$ with $u^{\prime}(0)=0, u(\tau)=0$ and $u(t)>0$ for $t \in\left[0, \tau\right.$ [ whenever $g(x) / x \rightarrow 0$ for $x \rightarrow 0^{+}$(compare with the first assumption in (3.6)) and liminf $\operatorname{in}_{x \rightarrow+\infty} g(x) / x$ is large enough.

In conclusion, we can state the following consequence of Theorem 3.2.

Corollary 3.1 (Positive solutions for the superlinear case) Let $g: \mathbb{R}^{+} \rightarrow \mathbb{R}^{+}:=$ $[0,+\infty)$ be a $C^{1}$-function with $g(0)=0, g(s)>0$ for $s>0$ and such that

$$
g^{\prime}(0)=0, \quad g^{\prime}(+\infty)=+\infty \quad \text { and } \quad \int_{1}^{+\infty} \frac{1}{g}<\infty .
$$

Then problem (3.4) has at least one positive and decreasing solution. 
Proof. It is obvious that under the assumptions of the corollary, both conditions (3.5) and (3.6) are satisfied. Moreover, the hypothesis of superlinear growth at infinity given by $g^{\prime}(+\infty)=+\infty$ implies the existence of sign-changing solutions.

Notice that even if we implicitly suppose that $g(s)$ is strictly increasing for large $s>0$, we do not assume global monotonicity.

Example 3.2 We refer here to the equivalent equation (2.3). Let $h: \mathbb{R}^{+} \rightarrow \mathbb{R}^{+}$be a continuous function, of class $C^{1}$ on $\mathbb{R}_{0}^{+}$, with $h(0)=0$ and $h(s)>0$ for $s>0$. If we suppose that

$$
\int_{0}^{+\infty} h=+\infty
$$

we have that the primitive $H(s)=\int_{0}^{s} h(\xi) d \xi$ is such that $H\left(\mathbb{R}_{0}^{+}\right)=\mathbb{R}_{0}^{+}$, with $H(s)$ strictly increasing. Recalling that $g^{\prime}(s)=h^{\prime}(s) / h(s)^{2}$, we conclude that $\left(f_{\infty}\right)$ and $\left(f_{0}\right)$ hold if and only if

$$
\left.\left.\lim _{s \rightarrow+\infty} \frac{h^{\prime}(s)}{h(s)^{2}}=0 \quad \text { and } \quad \lim _{s \rightarrow 0^{+}} \frac{h^{\prime}(s)}{h(s)^{2}} \in\right] 0,+\infty\right] .
$$

As a next step, we consider the primitive $F(x)=\int_{1}^{x} f(\xi) d \xi$ for $f(x)=g^{\prime}\left(H^{-1}(x)\right)$. The same computation as in Example 3.1 yields to

$$
\begin{aligned}
F(x) & =\int_{1}^{x} g^{\prime}\left(H^{-1}(\xi)\right) d \xi=\int_{c}^{H^{-1}(x)} g^{\prime}(u) h(u) d u=\int_{c}^{H^{-1}(x)} \frac{h^{\prime}(u)}{h(u)} d u \\
& =\log \left(h\left(H^{-1}(x)\right)\right)-K,
\end{aligned}
$$

for $c:=H^{-1}(1)$ and $K:=\log h(c)$. As a consequence,

$$
F(+\infty)=\log (h(+\infty))-K
$$

and thus $\left(F_{\infty}\right)$ holds if and only if

$$
\liminf _{s \rightarrow+\infty} h(s)>0 .
$$

We plan to apply Theorem 3.1 and therefore we look for an equivalent formulation of $(A F)$ in the above setting. Actually one can easily check that $(A F)$ holds if and only if there exists $\theta>0$ such that

$$
\lim _{t \rightarrow 0^{+}} \frac{h\left(H^{-1}((1+\theta) t A(t))\right)}{A(t)}=0^{+} .
$$


To produce our corollary, we also consider the simplifying assumption (3.3). In such a situation, $(A F)$ is satisfied if and only if

$$
\lim _{s \rightarrow 0^{+}} \frac{h(s)^{\alpha+1}}{H(s)^{\alpha}}=0^{+} .
$$

In conclusion, we can state the following consequence of Theorem 3.1.

Corollary 3.2 (Positive solutions for the singular case) Let $h: \mathbb{R}_{0}^{+} \rightarrow \mathbb{R}_{0}^{+}$be a $C^{1}$-function with $h\left(0^{+}\right)=0$. Assume (3.7), (3.8), (3.9) and (3.10). If we further suppose that a(t) satisfies (3.3), then the equation

$$
u^{\prime \prime}=\frac{a(t)}{h(u)}
$$

has at least one (positive) increasing solution satisfying the Neumann boundary conditions.

An easy case for the validity of Corollary 3.2 is when $h(s)=s^{\sigma}$ for $\sigma>\alpha$. Note that if $\lim _{t \rightarrow 0^{+}} a(t)=c>0$, we have $\alpha=1$ and we obtain the strong force condition $\sigma>1$. This assumption, so named after the pioneering work by Gordon [22], has been widely considered when studying dynamical systems with singularities. In this area, a great deal of existence and multiplicity results have been achieved for second order scalar equations, as well as for Hamiltonian systems, using variational or topological methods. However, most of the research in this direction has concerned equations with a different structure, typically, autonomous equations with a singularity of attractive or repulsive type, perturbed by an external forcing term (see, for instance, [15] and the references therein). Thus, a simple model like $u^{\prime \prime}=a(t) / u^{\sigma}$ seems to represent a quite new topic of investigation (and, to the best of our knowledge, only few recent contributions as $[12,16,35]$ can be quoted).

Example 3.3 Let $g: \mathbb{R} \rightarrow \mathbb{R}_{0}^{+}$be a $C^{1}$-function. If we suppose that

$$
\int_{-\infty}^{0} \frac{1}{g}=+\infty, \quad \int_{0}^{+\infty} \frac{1}{g}<+\infty
$$

we can take a primitive $H$ of $-1 / g$ such that $H(\mathbb{R})=\mathbb{R}_{0}^{+}$. Note that $H(s)$ is a strictly decreasing function with $H(-\infty)=+\infty$ and $H(+\infty)=0^{+}$, that is, a strictly decreasing diffeomorphism of $\mathbb{R}$ onto $\mathbb{R}_{0}^{+}$. Accordingly $\left(f_{\infty}\right)$ and $\left(f_{0}\right)$ hold if and only if

$$
\left.\left.g^{\prime}(-\infty)=0 \quad \text { and } \quad g^{\prime}(+\infty) \in\right] 0,+\infty\right]
$$


As a next step, we consider the primitive $F(x)=\int_{1}^{x} f(\xi) d \xi$ for $f(x)=g^{\prime}\left(H^{-1}(x)\right)$. The same computation as in Example 3.1 yields

$$
F(x)=K-\log \left(g\left(H^{-1}(x)\right)\right),
$$

for $c:=H^{-1}(1)$ and $K:=\log g(c)$. As a consequence

$$
F(+\infty)=-\log (g(-\infty))
$$

and thus $\left(F_{\infty}\right)$ holds if and only if

$$
\limsup _{s \rightarrow-\infty} g(s)<+\infty .
$$

We plan to apply Theorem 3.1 and therefore we discuss now assumption $(A F)$. Similarly as in Example 3.2, it is easy to check that $(A F)$ holds if and only if there exists $\theta>0$ such that

$$
\lim _{t \rightarrow 0^{+}} g\left(H^{-1}((1+\theta) t A(t))\right) A(t)=+\infty .
$$

Moreover, if we assume that $a(t)$ satisfies (3.3), then $(A F)$ is fulfilled if and only if

$$
\lim _{s \rightarrow+\infty} g(s)^{\alpha+1} H(s)^{\alpha}=+\infty .
$$

In conclusion, we can state the following consequence of Theorem 3.1.

Corollary 3.3 (Exponential type nonlinearities) Let $g: \mathbb{R} \rightarrow \mathbb{R}_{0}^{+}$be a $C^{1}$-function such that (3.11), (3.12), (3.13) and (3.14) hold true. If we further suppose that a ( $t)$ satisfies (3.3), then problem (3.4) has at least one decreasing solution.

An easy case for Corollary 3.3 is for $g(s)=\exp (s)$. In this situation, however, a better result can be proved, since we are in the setting of (3.2) and we do not need the assumption (3.3) on the weight function.

We conclude this section with a description of the argument which is used in the proof of our main result concerning the Neumann boundary value problem (3.1). For the next discussion, we recall that the weight function $a(t)$ is assumed to satisfy conditions $\left(A_{0}\right),\left(A_{1}\right)$ and $\left(A_{2}\right)$.

First of all, we consider the equivalent first order planar system in the open domain $\mathcal{D}:=\mathbb{R}_{0}^{+} \times \mathbb{R}$,

$$
\left\{\begin{array}{l}
x^{\prime}=y \\
y^{\prime}=f(x) y^{2}+a(t),
\end{array}\right.
$$


that we also write in a compact form as

$$
z^{\prime}=Z(t, z), \quad z=(x, y) \in \mathcal{D} .
$$

For each $z_{0} \in \mathcal{D}$ and $s \in[0, T]$, we denote by $\zeta\left(\cdot ; s, z_{0}\right)$ the solution of (3.15) with $\zeta(s)=z_{0}$ and, for every $\sigma \in[0, T]$ such that $\zeta\left(\sigma ; s, z_{0}\right)$ is defined, we denote by

$$
\Phi_{s}^{\sigma}: z_{0} \mapsto \zeta\left(\sigma ; s, z_{0}\right)
$$

the Poincaré map which maps any initial point $z_{0}$ to the point reached by the solution at the time $\sigma$.

We consider at first a maximal open interval $\left.] r_{0},+\infty\right)$, with $r_{0} \geq 0$, such that for every point $P=(r, 0)$, with $r>r_{0}$, the solution $\zeta(t ; 0, P)$ is defined for all $t \in[0, \tau]$. From the assumption $a(t) \geq 0$ for $t \in[0, \tau]$, it follows that the set

$$
\mathcal{D}^{+}:=\{(x, y) \in \mathcal{D}: y \geq 0\}
$$

is positively invariant for the solutions of (3.15) along the time-interval $[0, \tau]$ (see, for instance, [11, Section 5.2]). From Lemma 2.3 and $\int_{0}^{\tau} a(t) d t>0$, it follows also that, for each $r>r_{0}$

$$
\Phi_{0}^{\tau}(r, 0) \in \mathcal{D}_{0}^{+}:=\{(x, y) \in \mathcal{D}: y>0\} .
$$

Thus, setting

$$
\Gamma_{0}:=\left\{\Phi_{0}^{\tau}(r, 0): r>r_{0}\right\}
$$

we have that $\Gamma_{0}$ is a curve contained in $\mathcal{D}_{0}^{+}$.

In a symmetrical manner we consider a maximal open interval $\left.] r_{1},+\infty\right)$, with $r_{1} \geq 0$, such that for every point $P=(r, 0)$, with $r>r_{1}$, the solution $\zeta(t ; T, P)$ is defined for all $t \in[\tau, T]$. In other words, we look for solutions which go backward in time in the interval $[\tau, T]$. From the assumption $a(t) \leq 0$ for $t \in[\tau, T]$, it follows that the set $\mathcal{D}^{+}$is negatively invariant for the solutions of (3.15) along the timeinterval $[\tau, T]$. From Lemma 2.4 and $\int_{\tau}^{T} a(t) d t<0$, it follows also that, for each $r>r_{1}$,

$$
\Phi_{T}^{\tau}(r, 0) \in \mathcal{D}_{0}^{+} .
$$

Thus, setting

$$
\Gamma_{1}:=\left\{\Phi_{T}^{\tau}(r, 0): r>r_{1}\right\}
$$

we have that $\Gamma_{1}$ is a curve contained in $\mathcal{D}_{0}^{+}$.

Suppose now that there exists an intersection point

$$
Q \in \Gamma_{0} \cap \Gamma_{1} .
$$


If this happens, it means that there exists a point $P=(\bar{r}, 0)$ with $\bar{r}>r_{0}$ such that the solution $\zeta(\cdot ; 0, P)$ is defined on $[0, \tau]$ with $\zeta(\tau ; 0, P)=Q$ and, moreover, $\zeta(T ; \tau, Q)=\left(\Phi_{T}^{\tau}\right)^{-1}(Q)=(\hat{r}, 0)$ for some $\hat{r}>r_{1}$. In conclusion the solution $\zeta(\cdot ; 0, P)$ is defined on $[0, T]$ with $x(0)=\bar{r}, x(T)=\hat{r}$ and $x^{\prime}(0)=x^{\prime}(T)=0$. Moreover, we have $x^{\prime}=y \geq 0$, that is $x(t)$ is an increasing solution of (3.1). Actually, from Lemma 2.2 one could prove that, unless $a(t)$ is identically zero in a neighborhood of $t=0$ or $t=T$, it holds that $x(t)$ is strictly increasing.

If instead of condition $\left(A_{2}\right)$ we assume the symmetric condition

$\left(A_{2}^{\prime}\right) \quad a(t) \leq 0$, for a.e. $t \in[0, \tau]$ and $a(t) \geq 0$, for a.e. $t \in[\tau, T]$,

via the same argument we can provide the existence of a decreasing solution to the problem (3.1).

The proof that the curves $\Gamma_{0}$ and $\Gamma_{1}$ actually intersect will be performed by means of three technical lemmas which are proved in the next section. The first one (see Lemma 4.1) asserts that if $f(+\infty)=0$, then for $r>0$ and sufficiently large, the $y$-component of $\Phi_{t_{0}}^{t_{1}}(r, 0)$ is near to $\int_{t_{0}}^{t_{1}} a(t) d t$. As a consequence, the hypothesis $\left(A_{1}\right)$ implies that for $r>0$ large enough, the points of the curve $\Gamma_{0}$ are between the $x$-axis and the points of $\Gamma_{1}$. On the other hand, under suitable assumptions on the growth of $f(x)$ as $x \rightarrow 0^{+}$we can prove that $\Gamma_{1}$ is bounded in the $y$-component and accumulates at zero in the $x$-component as $r \rightarrow r_{1}^{+}$(see Lemma 4.3), while $\Gamma_{0}$ becomes unbounded in the $y$-component as $r \rightarrow r_{0}^{+}$(see Lemma 4.2). A possible behavior of the curves $\Gamma_{0}$ and $\Gamma_{1}$ is illustrated in Figure 1.

If the integral condition $\left(A_{1}\right)$ is not satisfied, we know that there are no solutions to (3.1) when $f>0$. In the framework of our approach, this means that the curves $\Gamma_{0}$ and $\Gamma_{1}$ will not intersect (see Figure 2 for a graphical representation of this situation). 


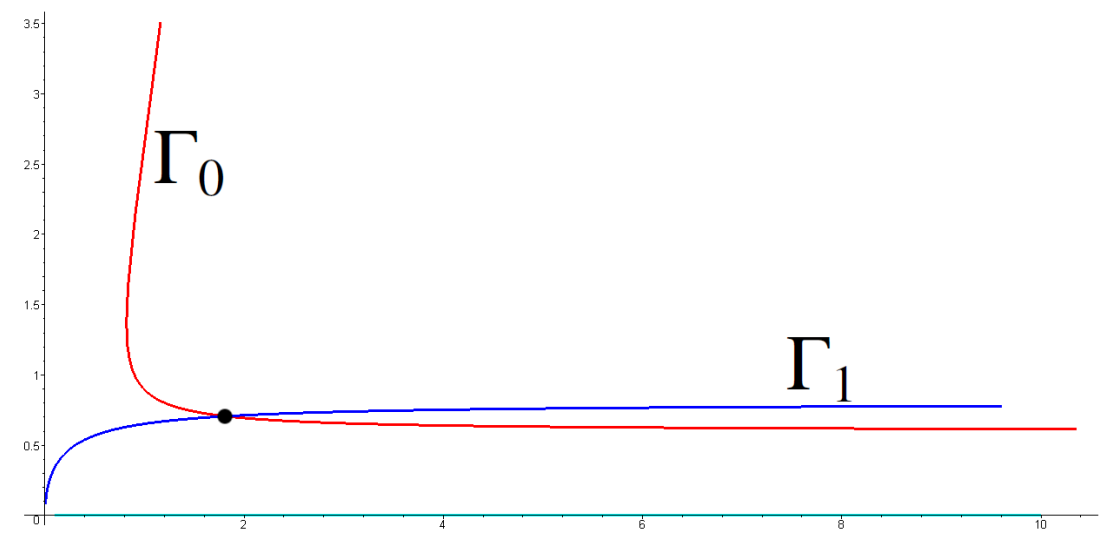

Figure 1: The present figure illustrates a typical behavior of the curves $\Gamma_{0}$ and $\Gamma_{1}$. We have marked the resulting intersection point. For this example we have taken $f(x)=1 / x$ on $\mathbb{R}_{0}^{+}$and as $a(t)$ a stepwise function with $a(t)=0.5$ on $[0, \tau]=[0,1.2]$ and $a(t)=-0.8$ on $[\tau, T]=[1.2,2.2]$. The resulting integral $\int_{0}^{T} a(t) d t$ is negative. One can also see that at the right-hand side of the graph the curve $\Gamma_{0}$ is close to the line $y=0.6$, while $\Gamma_{1}$ is close to the line $y=0.8$, consistently with Lemma 4.1 .

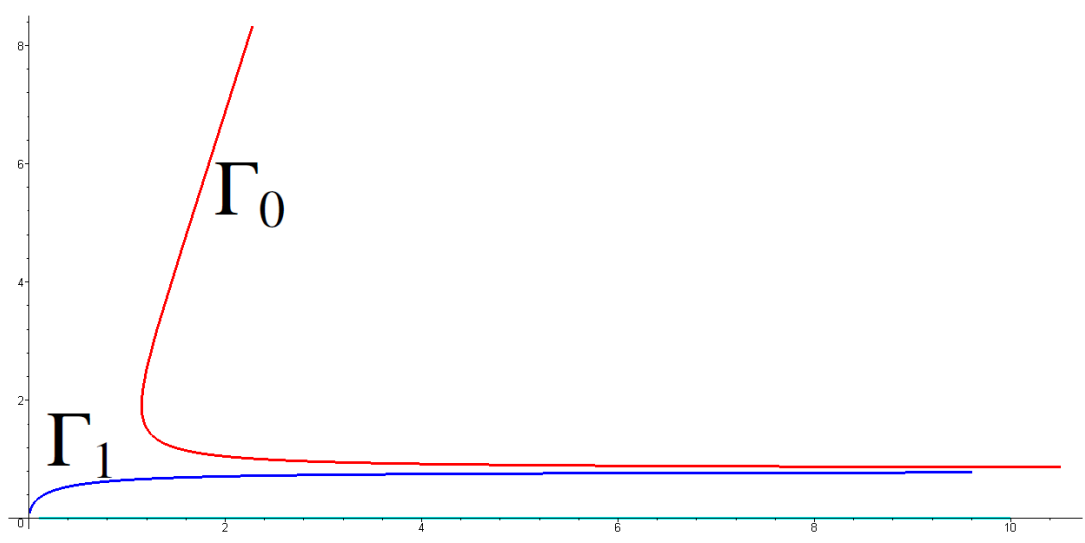

Figure 2: The present figure illustrates a possible behavior of the curves $\Gamma_{0}$ and $\Gamma_{1}$ when $f>0$ and the integral condition $\left(A_{1}\right)$ is not satisfied. For this example we have taken $f(x)=1 / x$ on $\mathbb{R}_{0}^{+}$and as $a(t)$ a stepwise function with $a(t)=0.7$ on $[0, \tau]=[0,1.2]$ and $a(t)=-0.8$ on $[\tau, T]=[1.2,2.2]$. The resulting integral $\int_{0}^{T} a(t) d t$ is positive. One can also see that at the right-hand side of the graph the curve $\Gamma_{0}$ is close to the line $y=0.84$, while $\Gamma_{1}$ is close to the line $y=0.8$, consistently with Lemma 4.1 . 


\section{Technical results and proof of Theorem 3.1}

This section is devoted to the proof of the main results. Let us consider the differential equation

$$
x^{\prime \prime}=f(x)\left(x^{\prime}\right)^{2}+a(t),
$$

where $f: \mathbb{R}_{0}^{+} \rightarrow \mathbb{R}$ is a continuous function and $a \in L^{1}(0, T)$. Equation (4.1) is equivalent to the planar system in the open domain $\mathcal{D}:=\mathbb{R}_{0}^{+} \times \mathbb{R}$,

$$
\left\{\begin{array}{l}
x^{\prime}=y \\
y^{\prime}=f(x) y^{2}+a(t)
\end{array}\right.
$$

that we also write in a compact form as

$$
z^{\prime}=Z(t, z), \quad z=(x, y) \in \mathcal{D} .
$$

As remarked in Section 2, we know that the uniqueness of the solutions to the Cauchy problems associated to (4.2) is guaranteed.

Lemma 4.1 Let $\left[t_{0}, t_{1}\right] \subseteq[0, T]$. Assume

$$
f(+\infty):=\lim _{x \rightarrow+\infty} f(x)=0 .
$$

Then, there exists $R>0$ such that, for each $r \geq R$, the solution $(x(t), y(t))$ of (4.2) satisfying the initial condition $\left(x\left(t_{0}\right), y\left(t_{0}\right)\right)=(r, 0)$ is defined on $\left[t_{0}, t_{1}\right]$. Moreover, for each $\varepsilon>0$ there exists $R_{\varepsilon} \geq R$ such that, if $r \geq R_{\varepsilon}$, it holds that

$$
\left|y\left(t_{1}\right)-\int_{t_{0}}^{t_{1}} a(t) d t\right| \leq \varepsilon
$$

and

$$
\left|x\left(t_{1}\right)-r\right| \leq N
$$

where $N$ is a fixed positive constant depending only on a $(t)$.

Proof. Let us define

$$
A_{t_{0}}(t):=\int_{t_{0}}^{t} a(\xi) d \xi, \quad \mathcal{A}(t):=\int_{t_{0}}^{t} A_{t_{0}}(\xi) d \xi .
$$

Suppose $A_{t_{0}}(t) \not \equiv 0$ (otherwise the result is trivial) and set $M:=\left|A_{t_{0}}\right|_{\infty}>0$. We choose a constant $\varepsilon^{\prime}>0$ such that

$$
2 M^{2} \varepsilon^{\prime}\left(t_{1}-t_{0}\right) \leq \frac{\pi}{4}
$$


In virtue of condition $\left(f_{\infty}\right)$, take a constant $K_{\mathcal{\varepsilon}^{\prime}}>0$ such that $|f(x)| \leq \varepsilon^{\prime}, \forall x \geq K_{\mathcal{\varepsilon}^{\prime}}$ and define

$$
H_{\mathcal{\varepsilon}^{\prime}}:=K_{\varepsilon^{\prime}}+4 M^{2} \varepsilon^{\prime}\left(t_{1}-t_{0}\right)^{2}+|\mathcal{A}|_{\infty}+1 .
$$

Suppose that $x\left(t_{0}\right)=r \geq H_{\varepsilon^{\prime}}$ and define $\left.\left.\beta \in\right] t_{0}, t_{1}\right]$ as the maximal value such that $(x(t), y(t))$ is defined and $x(t)>K_{\varepsilon^{\prime}}$ for all $t \in\left[t_{0}, \beta[\right.$. The position

$$
x(t)=u(t)+\mathcal{A}(t)
$$

transforms equation (4.1) into the equivalent equation

$$
u^{\prime \prime}=f(u+\mathcal{A}(t))\left(u+A_{t_{0}}(t)\right)^{2},
$$

which can be written as the first order system

$$
\left\{\begin{array}{l}
u^{\prime}=v \\
v^{\prime}=f(u+\mathcal{A}(t))\left(v+A_{t_{0}}(t)\right)^{2},
\end{array}\right.
$$

in the $(u, v)$-plane. From the second equation in system (4.6), we get

$$
\left|v^{\prime}(t)\right| \leq 2|f(u(t)+\mathcal{A}(t))|\left(v(t)^{2}+M^{2}\right) \leq 2 \varepsilon^{\prime}\left(v(t)^{2}+M^{2}\right), \quad \forall t \in\left[t_{0}, \beta[,\right.
$$

that is,

$$
\left|\frac{v^{\prime}(t)}{v(t)^{2}+M^{2}}\right| \leq 2 \varepsilon^{\prime}, \quad \forall t \in\left[t_{0}, \beta[\right.
$$

An integration gives

$$
|v(t)| \leq M \tan \left(2 M \varepsilon^{\prime}\left(t_{1}-t_{0}\right)\right) \leq 4 M^{2} \varepsilon^{\prime}\left(t_{1}-t_{0}\right),
$$

for all $t \in\left[t_{0}, \beta\right.$ (here we use the fact that $|\tan (x)| \leq 2|x|$ for $|x| \leq \pi / 4$ and recall (4.5)). From the bound (4.7), we immediately find that

$$
|u(t)-r|=\left|u(t)-u\left(t_{0}\right)\right| \leq 4 M^{2} \varepsilon^{\prime}\left(t_{1}-t_{0}\right)^{2}
$$

holds for all $t \in\left[t_{0}, \beta[\right.$. As a consequence

$$
x(t)=u(t)-r+r+\mathcal{A}(t) \geq-4 M^{2} \varepsilon^{\prime}\left(t_{1}-t_{0}\right)^{2}+H_{\mathcal{\varepsilon}^{\prime}}-|\mathcal{A}|_{\infty}=K_{\varepsilon^{\prime}}+1,
$$

for every $t \in\left[t_{0}, \beta\left[\right.\right.$. Then, the maximality of $\beta$ implies that $\beta=t_{1}$ and the bounds (4.7)-(4.8) on $\left[t_{0}, \beta\left[=\left[t_{0}, t_{1}\left[\right.\right.\right.\right.$ imply that $(x(t), y(t))$ is defined for $t=t_{1}$ and (4.7)(4.8) are satisfied for all $t \in\left[t_{0}, t_{1}\right]$. The first part of the Lemma is thus proved with the choice $R=H_{\varepsilon^{\prime}}$. 
For the second part of the Lemma, we just observe that $v(t)=y(t)-\int_{t_{0}}^{t} a(\xi) d \xi$ and, in view of (4.8),

$$
\left|x\left(t_{1}\right)-r\right| \leq\left|u\left(t_{1}\right)-r\right|+|\mathcal{A}|_{\infty} \leq 4 M^{2} \varepsilon^{\prime}\left(t_{1}-t_{0}\right)^{2}+|\mathcal{A}|_{\infty} .
$$

Hence, (4.3) and (4.4) follow from (4.7) and (4.9), with the choice $R_{\varepsilon}=H_{\varepsilon^{\prime}}$ and $\varepsilon^{\prime}>0$ so small that the inequalities

$$
4 M^{2} \varepsilon^{\prime}\left(t_{1}-t_{0}\right) \leq \epsilon, \quad 4 M^{2} \varepsilon^{\prime}\left(t_{1}-t_{0}\right)^{2} \leq 1
$$

are satisfied. For the constant $N$ (which must depend only on $a(t)$ ) we can take $N:=|\mathcal{A}|_{\infty}+1$.

In the sequel, the hypotheses $\left(A_{0}\right) \&\left(A_{2}\right)$ on the coefficient $a(t)$ will be summarized into the following condition that includes (and is equivalent to) both of them:

$\left(A_{3}\right) \quad a(t) \geq 0, a(t) \not \equiv 0$ on $[0, \tau]$ and $\quad a(t) \leq 0, a(t) \not \equiv 0$ on $[\tau, T]$.

Since we are interested in the search of solutions to (4.1) satisfying the Neumann boundary conditions

$$
x^{\prime}(0)=x^{\prime}(T)=0,
$$

it is not restrictive if we suppose that $a(t) \not \equiv 0$ on a right neighborhood of $t=0$ and on a left neighborhood of $t=T$ (otherwise, if $a \equiv 0$ on $\left[0, \sigma_{0}\right]$ and on $\left[\sigma_{1}, T\right]$, it will be sufficient to consider the Neumann boundary conditions on $\left[\sigma_{0}, \sigma_{1}\right]$ and then extend the solution to $[0, T]$ by taking constant values on $\left[0, \sigma_{0}\right]$ and on $\left.\left[\sigma_{1}, T\right]\right)$.

We now follow the strategy described at the end of Section 3 to which we refer for the notation used in the following. We also recall the positions

$$
F(x):=\int_{1}^{x} f(\xi) d \xi, \quad A(t):=\int_{0}^{t} a(\xi) d \xi .
$$

Applying Lemma 4.1 on the interval $[0, \tau]$, we know that, as a consequence of $\left(f_{\infty}\right)$, there exists a minimal $r_{0} \geq 0$ such that for all $r>r_{0}$, the solution $\zeta(t ; 0,(r, 0))$ of system (4.2) with $\zeta(0)=(r, 0)$ is defined on $[0, \tau]$. Moreover, assuming (without loss of generality) that in any right neighborhood of $t=0$ there exists some $t$ such that $a(t)>0$, we find that $\zeta(t) \in \mathcal{D}_{0}^{+}$for all $\left.\left.t \in\right] 0, \tau\right]$.

Lemma 4.2 Assume (besides $\left.\left(f_{\infty}\right)\right)$ that

$$
\left.\left.f\left(0^{+}\right):=\lim _{x \rightarrow 0^{+}} f(x) \in\right] 0,+\infty\right]
$$


and

$\left(F_{\infty}\right) \quad \liminf _{x \rightarrow+\infty} F(x)>-\infty$.

Moreover, let us suppose that there exists $\theta>0$ such that

$$
\lim _{t \rightarrow 0^{+}} \frac{\exp [F((1+\theta) t A(t))]}{A(t)}=0^{+} .
$$

Then the curve $\Gamma_{0}$ is unbounded in its y-component as $r \rightarrow r_{0}^{+}$.

Proof. Recall that we work in the interval $[0, \tau]$ where $a(t) \geq 0$ for a.e. $t$ and, moreover, $A(t)>0$ for all $t \in] 0, \tau]$. In order to simplify the notation, along the proof we denote by $\left(x_{r}(t), y_{r}(t)\right)$ the solution $\zeta(t ; 0,(r, 0))$. Accordingly, the Poincare map on $[0, \tau]$ is expressed as $\Phi_{0}^{\tau}(r, 0)=\left(x_{r}(\tau), y_{r}(\tau)\right)$. We want to prove that

$$
\lim _{r \rightarrow r_{0}^{+}} y_{r}(\tau)=+\infty .
$$

This implies that the curve $\Gamma_{0}$ is unbounded in the $y$-component. We distinguish two cases: $r_{0}>0$ and $r_{0}=0$.

Suppose that $r_{0}>0$. Assume, by contradiction that there exist a sequence $r_{n} \rightarrow r_{0}^{+}($as $n \rightarrow \infty)$ and a constant $M>0$ such that $y_{r_{n}}(\tau) \in[0, M]$. Since $x^{\prime}=y \geq 0$, we have that

$$
r_{0} \leq r_{n} \leq x_{r_{n}}(t) \leq x_{r_{n}}(\tau)
$$

According to $\left(F_{\infty}\right)$, let $L>0$ be such that $F(x) \geq-L$, for all $x \geq r_{0}$. From

$$
\frac{d}{d t}(y(t) \exp (-F(x(t))))=a(t) \exp (-F(x(t))) \geq 0, \quad \text { for a.e. } t \in[0, \tau]
$$

(compare with (2.8)), we find that the map $y(t) \exp (-F(x(t)))$ is nondecreasing on $[0, \tau]$ and therefore

$$
\begin{aligned}
0 \leq y_{r_{n}}(t) & =x_{r_{n}}^{\prime}(t) \leq y_{r_{n}}(\tau) \exp \left(F\left(x_{r_{n}}(t)\right)-F\left(x_{r_{n}}(\tau)\right)\right) \\
& \leq M \exp (L) \exp \left(F\left(x_{r_{n}}(t)\right)\right) .
\end{aligned}
$$

Hence,

$$
\mathscr{F}\left(x_{r_{n}}(t)\right)-\mathscr{F}\left(r_{n}\right) \leq \tau M \exp (L), \quad \forall t \in[0, \tau],
$$

for $\mathscr{F}(x)$ a primitive of $\exp (-F(x))$. From this we easily obtain a uniform bound for $x_{r_{n}}$ of the form

$$
r_{0} \leq x_{r_{n}}(t) \leq x_{r_{n}}(\tau) \leq N, \quad \forall t \in[0, \tau],
$$


where $N>0$ is a suitable constant independent on $n$. By the bound on $x_{r_{n}}$ and (4.11) we also get a uniform bound for $y_{r_{n}}$. A standard use of the Ascoli-Arzelà theorem (via equation (4.2)) gives a subsequence of $\left(x_{r_{n}}(t), y_{r_{n}}(t)\right)$ converging uniformly on $[0, \tau]$ to a solution (actually the solution) of (4.2) with $(x(0), y(0))=\left(r_{0}, 0\right)$ and, of course, such a solution is defined on $[0, \tau]$. This is a contradiction to $r_{0}>0$. Indeed, by the minimality of $r_{0}$ we know that $\zeta\left(t ; 0,\left(r_{0}, 0\right)\right)$ is not defined on $[0, \tau]$.

Henceforth, we suppose that $r_{0}=0$. This means that for every $r>0$ the solution $\zeta(t ; 0,(r, 0))=\left(x_{r}(t), y_{r}(t)\right)$ is defined for all $t \in[0, \tau]$. Sometimes, when no confusion can occur, the subscript $r$ will be omitted. A common feature of all these solutions is that $r<x(t)$ and $y(t)>0$ for all $t>0$ and, moreover, $x(t)$ is strictly increasing. In order to prove (4.10), without loss of generality we can suppose that $0<r<\varepsilon_{0}$, where $] 0, \varepsilon_{0}$ ] is a suitably chosen interval where we suppose that $f(x) \geq 0$. Without loss of generality (by taking a smaller $\varepsilon_{0}$ if necessary), we can also assume that

$$
\varepsilon_{0}<d_{0}:=\int_{0}^{\tau}\left(\int_{0}^{t} a(\xi) d \xi\right) d t
$$

As a preliminary observation, we notice that for every choice of $r>0$ it holds that $x(\tau)>\varepsilon_{0}$. Indeed, the result is obvious if $x(0)=r \geq \varepsilon_{0}$. On the other hand, if, by contradiction, we suppose that $x(t) \leq \varepsilon_{0}$ for all $t \in[0, \tau]$, then $x^{\prime \prime}(t)=$ $f(x(t))\left(x^{\prime}(t)\right)^{2}+a(t) \geq a(t)$ for a.e. $t \in[0, \tau]$ and hence

$$
\varepsilon_{0} \geq x(\tau)=r+\int_{0}^{\tau} x^{\prime}(t) d t \geq d_{0}
$$

(a contradiction).

Thanks to this observation, if we define

$$
-K:=\inf \left\{F(x): x \geq \varepsilon_{0}\right\},
$$

from $\left(F_{\infty}\right)$, we know that $-K>-\infty$ and

$$
F(x(\tau)) \geq-K,
$$

for each solution $x(\cdot)=x_{r}(\cdot)$ (with $r>0$.).

Let $r>0$ small. Since the map $s \mapsto \theta s A(s)$ (with $\theta>0$ the constant appearing in $(A F)$ ) is strictly increasing on a right neighborhood of $s=0$, we have $r=\theta s A(s)$ for a unique $s>0$ small. Henceforth, we assume that $s A(s)<\varepsilon_{0} /(1+\theta)$. We claim that there exists $t \in] 0, s]$ such that $y(t) \geq A(s)$. Indeed, if $y(t)<A(s)$ for every $t \in[0, s]$, then

$$
x(t)=r+\int_{0}^{t} y(\xi) d \xi \leq r+t A(s) \leq r+s A(s)=(1+\theta) s A(s)<\varepsilon_{0}, \quad \forall t \in[0, s] .
$$


Hence $y^{\prime}(t)=f(x(t)) y(t)^{2}+a(t) \geq a(t)$ for a.e. $t \in[0, s]$, which implies $y(s) \geq A(s)$, a contradiction. We can now define $\sigma \leq s$ as the first instant such that $y(\sigma)=A(s)$. Then $y(t)<A(s)$ for every $t \in[0, \sigma[$ so that

$$
\theta s A(s)=r \leq x(\sigma) \leq r+\sigma A(s) \leq r+s A(s)=(1+\theta) s A(s) .
$$

On the other hand, for a.e. $t>0$, we have

$$
\frac{y^{\prime}(t)}{y(t)}=f(x(t)) x^{\prime}(t)+\frac{a(t)}{y(t)} \geq f(x(t)) x^{\prime}(t) .
$$

Hence integrating on $[\sigma, \tau]$ and using (4.12) we obtain

$$
\log (y(\tau)) \geq \log (y(\sigma))+F(x(\tau))-F(x(\sigma)) \geq \log (A(s))-K-F(\xi),
$$

where $\xi:=x(\sigma) \in[r, r+s A(s)]=[\theta s A(s),(1+\theta) s A(s)]$. The function $F$ is nondecreasing on the interval $[\theta s A(s),(1+\theta) s A(s)] \subseteq] 0, \varepsilon_{0}[$ and therefore, from the above inequality, we obtain

$$
y(\tau) \geq \exp (-K) \frac{A(s)}{\exp [F((1+\theta) s A(s))]} .
$$

Hence, by assumption $(A F)$ we conclude that $y(\tau)=y_{r}(\tau) \rightarrow+\infty$ as $r=\theta s A(s) \rightarrow$ $0^{+}$and thus (4.10) holds.

Remark 4.1 A careful reading of the proof of Lemma 4.2 shows that $\left(f_{0}\right)$ could be replaced by the weaker hypothesis that $f(x) \geq 0$ for an interval of the form $\left.] 0, \varepsilon_{0}\right]$. Note also that we do not need to assume neither $\left(f_{0}\right)$ nor $(A F)$ if we know that $r_{0}>0$, which means that there exists a solution $\zeta(t ; 0,(r .0))$ which is not globally defined on $[0, \tau]$. In fact, $\left(f_{0}\right)$ and $(A F)$ were used only in the second part of the proof where $r_{0}=0$.

Applying now Lemma 4.1 on the interval $[\tau, T]$ (and reversing time) we find (as a consequence of $\left.\left(f_{\infty}\right)\right)$ a minimal $r_{1} \geq 0$ such that for all $r>r_{1}$, the solution $\zeta(t ; T,(r, 0))$ of system $(4.2)$ with $\zeta(T)=(r, 0)$ is defined on $[\tau, T]$. Moreover, assuming (without loss of generality) that in any left neighborhood of $t=T$ there exists some $t$ such that $a(t)<0$, we find that $\zeta(t) \in \mathcal{D}_{0}^{+}$for all $t \in[\tau, T[$.

Lemma 4.3 Assume (besides $\left(f_{\infty}\right)$ ) that

$$
\limsup _{x \rightarrow 0^{+}} F(x)<+\infty .
$$

Then the curve $\Gamma_{1}$ is bounded in its y-component and accumulates at 0 in its $x$ component, as $r \rightarrow r_{1}^{+}$. 
Proof. Recall that we work in the interval $[\tau, T]$ where $a(t) \leq 0$ for a.e. $t$ and, moreover, $\int_{t}^{T} a(\xi) d \xi<0$ for all $t \in[\tau, T$ [. Again, in order to simplify the notation, along the proof we denote by $\left(x_{r}(t), y_{r}(t)\right)$ the solution $\zeta(t ; T,(r, 0))$. Accordingly, the backward Poincaré map on $[\tau, T]$ is expressed as $\Phi_{T}^{\tau}(r, 0)=\left(x_{r}(\tau), y_{r}(\tau)\right)$ (sometimes, when no confusion can occur, the subscript $r$ will be omitted). We want to prove that

$$
\lim _{r \rightarrow r_{1}^{+}} x_{r}(\tau)=0^{+}, \quad \limsup _{r \rightarrow r_{1}^{+}} y_{r}(\tau)<+\infty .
$$

We recall that all such solutions satisfy $x(t)<r$ and $y(t)>0$ for all $t<T$ and, moreover, $x(t)$ is strictly increasing.

We first prove that $\lim \sup _{r \rightarrow r_{1}^{+}} y_{r}(\tau)<+\infty$. According to $\left(F_{0}\right)$, take $M>0$ such that $F(x) \leq M$ for $\left.x \in] 0, r_{1}+1\right]$ and define

$$
K:=|a|_{1}+M+\left|F\left(r_{1}+1\right)\right|+\int_{r_{1}+1}^{r_{1}+2}|f(\xi)| d \xi, \quad L:=\exp (K)+1 .
$$

We show that $y(\tau) \leq L$ for every $\left.r \in] r_{1}, r_{1}+2\right]$. Assume by contradiction that there is $t \in\left[\tau, T\right.$ [ such that $y(t)>L$. Then we can find a maximal interval $\left[t_{0}, t_{1}\right] \subset[\tau, T]$ such that $y\left(t_{0}\right)=L, y\left(t_{1}\right)=1$ and $1<y(t)<L$ for $\left.t \in\right] t_{0}, t_{1}[$. Since

$$
\left.\frac{y^{\prime}(t)}{y(t)}=f(x(t)) x^{\prime}(t)+\frac{a(t)}{y(t)} \geq f(x(t)) x^{\prime}(t)-|a(t)|, \quad \text { for a.e. } t \in\right] t_{0}, t_{1}[,
$$

an integration on $\left[t_{0}, t_{1}\right]$ gives

$$
\log (L)=\log \left(y\left(t_{0}\right)\right) \leq-\int_{x\left(t_{0}\right)}^{x\left(t_{1}\right)} f(\xi) d \xi+|a|_{1} .
$$

If $x\left(t_{0}\right) \geq r_{1}+1$, we have $-\int_{x\left(t_{0}\right)}^{x\left(t_{1}\right)} f(\xi) d \xi \leq \int_{r_{1}+1}^{r_{1}+2}|f(\xi)| d \xi$; if $x\left(t_{0}\right)<r_{1}+1$, we have

$$
-\int_{x\left(t_{0}\right)}^{x\left(t_{1}\right)} f(\xi) d \xi \leq M-F\left(r_{1}+1\right)+\int_{r_{1}+1}^{r_{1}+2}|f(\xi)| d \xi
$$

In any case $\log (L) \leq K$, from which $L \leq \exp (K)$, a contradiction.

We now prove that $\lim _{r \rightarrow r_{1}^{+}} x_{r}(\tau)=0^{+}$. If $r_{1}=0$, the thesis immediately follows, since $x_{r}(\tau)<r$. Hence, we have to consider the case $r_{1}>0$. Assume by contradiction that there exists a sequence $r_{n} \rightarrow r_{1}^{+}($as $n \rightarrow \infty)$ and a constant $\varepsilon>0$ such that $x_{r_{n}}(\tau) \geq \varepsilon$. Since $x_{r_{n}}$ is strictly increasing, we have the uniform bound

$$
\varepsilon \leq x_{r_{n}}(t) \leq r_{n} \leq r_{1}+1
$$

Arguing similarly as in the proof of Lemma 4.2 (compare with (4.11)) and since $y_{r_{n}}(\tau)$ is bounded (as shown in the first part of this Lemma), we immediately obtain 
a uniform bound for $y_{r_{n}}$. We conclude again as in the proof of Lemma 4.2 (using Ascoli-Arzelà theorem) contradicting the minimality of $r_{1}>0$.

After these technical lemmas we are ready to conclude with the proof of our main results. The proof just casts in a formal style the informal argument described at the end of Section 3 (see also Figure 1).

Proof of Theorem 3.1. Along the proof, we denote by $\Pi_{x}:(x, y) \mapsto x$ and $\Pi_{y}$ : $(x, y) \mapsto y$ the projections of $\mathbb{R}^{2}$ onto its factors. We also set

$$
d^{-}:=\int_{\tau}^{T}|a(t)| d t, \quad d^{+}:=\int_{0}^{\tau} a(t) d t, \quad d_{0}:=\frac{d^{-}+d^{+}}{2} .
$$

By $\left(A_{0}\right)$ and $\left(A_{1}\right)$ we have that $0<d^{+}<d^{-}$.

Consider in $\mathcal{D}_{0}^{+}$the curves $\left.\left.\Gamma_{0}:=\Phi_{0}^{\tau}(] r_{0},+\infty\right) \times\{0\}\right)$ and $\left.\Gamma_{1}:=\Phi_{T}^{\tau}(] r_{1},+\infty\right) \times$ $\{0\})$, for $r_{0}, r_{1} \geq 0$ defined before.

Let $M_{1}>d^{-}+1$ be such that (according to Lemma 4.3)

$$
0<\Pi_{y}(x, y)<M_{1}, \quad \forall(x, y) \in \Gamma_{1},
$$

that is

$$
0<\Pi_{y}\left(\Phi_{T}^{\tau}(r, 0)\right)<M_{1}, \quad \forall r>r_{1} .
$$

We also take $0<\varepsilon_{1}<\min \left\{d^{+}, 1\right\}$ such that

$$
\varepsilon_{1}<\frac{d^{-}-d^{+}}{4}
$$

and, according to Lemma 4.1, we can find a (common) constant $R^{*}$ such that $\Phi_{0}^{\tau}(r, 0)$ and $\Phi_{T}^{\tau}(r, 0)$ are defined for every $r \geq R^{*}$ and, moreover

$$
\begin{aligned}
& 0<d^{+}-\varepsilon_{1} \leq \Pi_{y}\left(\Phi_{0}^{\tau}(r, 0)\right) \leq d^{+}+\varepsilon_{1}<d_{0}-\varepsilon_{1}< \\
& <d_{0}+\varepsilon_{1}<d^{-}-\varepsilon_{1} \leq \Pi_{y}\left(\Phi_{T}^{\tau}(r, 0)\right) \leq d^{-}+\varepsilon_{1}<M_{1} .
\end{aligned}
$$

For the second part of Lemma 4.1 we also know that, for a suitable choice of the constant $N$ (which must be well chosen both for the intervals $[0, \tau]$ and $[\tau, T]$ ), we also have

$$
r-N \leq \Pi_{x}\left(\Phi_{0}^{\tau}(r, 0)\right) \leq r+N, \quad r-N \leq \Pi_{x}\left(\Phi_{T}^{\tau}(r, 0)\right) \leq r+N .
$$

For instance, a suitable choice for $N$ can be given by $N:=T|a|_{L^{1}(0, T)}+1$. Once we have fixed the constants $M_{1}, \varepsilon_{1}, R^{*}$ and $N$, we proceed as follows. 
By Lemma 4.2 (see (4.10)) we know that $\Pi_{y}\left(\Phi_{0}^{\tau}(r, 0)\right) \rightarrow+\infty$ as $r \rightarrow r_{0}^{+}$. On the other hand, $\Pi_{y}\left(\Phi_{0}^{\tau}(r, 0)\right)<M_{1}$ for every $r \geq R^{*}$. Hence (by continuity), we find $\left.\alpha_{0} \in\right] r_{0}, R^{*}[$ such that

$$
\Pi_{y}\left(\Phi_{0}^{\tau}\left(\alpha_{0}, 0\right)\right)=M_{1} \quad \text { and } \quad \Pi_{y}\left(\Phi_{0}^{\tau}(r, 0)\right)<M_{1}, \forall r>\alpha_{0} .
$$

Next, we fix a constant $\eta^{+}$such that

$$
\eta^{+}>\delta_{\max }:=\max \left\{\Pi_{x}\left(\Phi_{0}^{\tau}(r, 0)\right): \alpha_{0} \leq r \leq R^{*}+N\right\}
$$

(note that $R^{*} \leq \delta_{\max }$ ). By the first estimate in (4.14) we know that $\Pi_{x}\left(\Phi_{0}^{\tau}(r, 0)\right)>\eta^{+}$ for each $r>\eta^{+}+N$. On the other hand, $\Pi_{x}\left(\Phi_{0}^{\tau}(r, 0)\right)<\eta^{+}$, for all $r \in\left[\alpha_{0}, R^{*}+N\right]$. Hence (by continuity), we find $\left.\beta_{0} \in\right] R^{*}+N, \eta^{+}+N[$ such that

$$
\Pi_{x}\left(\Phi_{0}^{\tau}\left(\beta_{0}, 0\right)\right)=\eta^{+} \quad \text { and } \Pi_{x}\left(\Phi_{0}^{\tau}(r, 0)\right)<\eta^{+}, \forall r \in\left[\alpha_{0}, \beta_{0}\right] .
$$

Now we can fix a constant $\eta^{-}$such that

$$
0<\eta^{-}<\delta_{\min }:=\min \left\{\Pi_{x}\left(\Phi_{0}^{\tau}(r, 0)\right): \alpha_{0} \leq r \leq \beta_{0}\right\} .
$$

With these settings, we can summarize the behavior of the curve $\Gamma_{0}$ across the rectangle $\mathcal{R}:=\left[\eta^{-}, \eta^{+}\right] \times\left[0, M_{1}\right]$ as follows:

$$
\begin{gathered}
\eta^{-}<\Pi_{x}\left(\Phi_{0}^{\tau}(r, 0)\right)<\eta^{+}, \quad \forall r \in\left[\alpha_{0}, \beta_{0}\left[\quad \text { and } \quad \Pi_{x}\left(\Phi_{0}^{\tau}\left(\beta_{0}, 0\right)\right)=\eta^{+}\right.\right. \\
\left.\left.\Pi_{y}\left(\Phi_{0}^{\tau}(r, 0)\right)<M_{1}, \quad \forall r \in\right] \alpha_{0}, \beta_{0}\right] \quad \text { and } \quad \Pi_{y}\left(\Phi_{0}^{\tau}\left(\alpha_{0}, 0\right)\right)=M_{1} .
\end{gathered}
$$

We pass now to consider the behavior of the curve $\Gamma_{1}$ across the rectangle $\mathcal{R}$. From Lemma 4.3 (see (4.13)) we know that $\Pi_{x}\left(\Phi_{T}^{\tau}(r, 0)\right) \rightarrow 0^{+}$as $r \rightarrow r_{1}^{+}$. On the other hand, the second estimate in (4.14) implies that $\Pi_{x}\left(\Phi_{T}^{\tau}(r, 0)\right)>\eta^{+}$for each $r>\eta^{+}+N$. Hence (by continuity), we can find $\alpha_{1}<\beta_{1}$ in $\left.] r_{1},+\infty\right)$ such that

$$
\left.\eta^{-}<\Pi_{x}\left(\Phi_{T}^{\tau}(r, 0)\right)<\eta^{+}, \quad \forall r \in\right] \alpha_{1}, \beta_{1}[
$$

and

$$
\Pi_{x}\left(\Phi_{T}^{\tau}\left(\alpha_{1}, 0\right)\right)=\eta^{-}, \quad \Pi_{x}\left(\Phi_{T}^{\tau}\left(\beta_{1}, 0\right)\right)=\eta^{+} .
$$

Moreover, by Lemma 4.3 and the choice of $M_{1}$ we also have

$$
\Pi_{y}\left(\Phi_{T}^{\tau}(r, 0)\right)<M_{1}, \quad \forall r \in\left[\alpha_{1}, \beta_{1}\right] .
$$

In order to conclude, consider the four points $P_{i}=\left(\hat{x}_{i}, \hat{y}_{1}\right)(i=1,2,3,4)$ on the boundary of $\mathcal{R}$

$$
P_{1}:=\Phi_{T}^{\tau}\left(\alpha_{1}, 0\right), P_{2}:=\Phi_{0}^{\tau}\left(\beta_{0}, 0\right), P_{3}:=\Phi_{T}^{\tau}\left(\beta_{1}, 0\right), P_{4}:=\Phi_{0}^{\tau}\left(\alpha_{0}, 0\right),
$$


for which we have

$$
\hat{x}_{1}=\eta^{-}, \hat{x}_{2}=\hat{x}_{3}=\eta^{+}, \eta^{-}<\hat{x}_{4}<\eta^{+}
$$

and

$$
0<\hat{y}_{1}<M_{1}, 0<\hat{y}_{2}<d_{0}<\hat{y}_{3}<M_{1}, \hat{y}_{4}=M_{1} .
$$

Consider also the $\operatorname{arcs} \Gamma_{0}^{\prime}$ and $\Gamma_{1}^{\prime}$ in $\mathcal{R}$

$$
\Gamma_{0}^{\prime}:=\left\{\Phi_{0}^{\tau}(r, 0): r \in\left[\alpha_{0}, \beta_{0}\right]\right\}, \quad \Gamma_{1}^{\prime}:=\left\{\Phi_{T}^{\tau}(r, 0): r \in\left[\alpha_{1}, \beta_{1}\right]\right\} .
$$

By construction, $\Gamma_{0}^{\prime}$ connects in $\mathcal{R}$ the point $P_{4}$ (on the upper side of $\mathcal{R}$ ) with $P_{2}$ (on the right-hand side of $\mathcal{R}$ ), while $\Gamma_{1}^{\prime}$ connects in $\mathcal{R}$ the point $P_{1}$ (on the left-hand side of $\mathcal{R}$ ) with $P_{3}$ (on the right-hand side of $\mathcal{R}$ ). Moreover, $P_{2}$ is below $P_{3}$ while $P_{4}$ is above $P_{1}$ (see Figure 3 ).

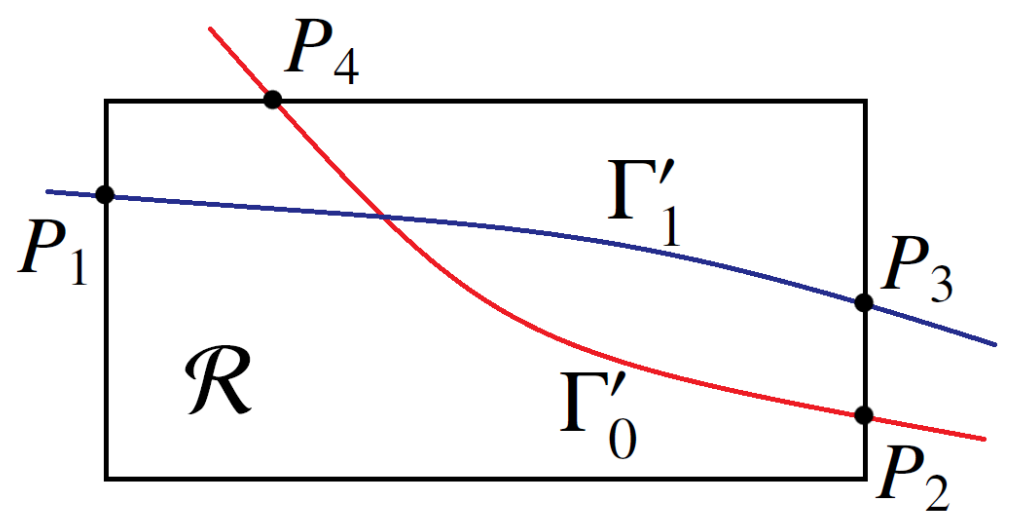

Figure 3: The present figure illustrates the conclusion of the proof of Theorem 3.1. The information we have is about the mutual position of the points on $\partial \mathcal{R}$ and the fact that the curves $\Gamma_{0}^{\prime}$ and $\Gamma_{1}^{\prime}$ (which are the restrictions of $\Gamma_{0}$ and $\Gamma_{1}$ with $\mathcal{R}$ ) connect pairs of opposite points. This is sufficient to guarantee the existence of a nonempty intersection for $\Gamma_{0}^{\prime} \cap \Gamma_{1}^{\prime}$.

Now, by an elementary argument of plane topology based on the Jordan Curve Theorem we can deduce that $\Gamma_{0}^{\prime} \cap \Gamma_{1}^{\prime} \neq \emptyset$ (see also [29] and [31] for applications of the same argument).

In order to check this claim, let us consider the curve $\mathscr{J}$, obtained by the union of the arc $\Gamma_{1}^{\prime}$ with the segments $\overline{\left[P_{1},\left(\eta^{-}, 0\right)\right]}, \overline{\left[\left(\eta^{-}, 0\right),\left(\eta^{+}, 0\right)\right]}$ and $\overline{\left[\left(\eta^{+}, 0\right), P_{3}\right]}$. By construction, $\mathscr{J}$ is a simple closed curve (a Jordan curve). Hence, a classical result of plane topology $[24, \S 13]$ asserts that $\mathbb{R}^{2} \backslash \mathscr{J}$ is the union of two (arcwise) connected open sets that we denote by $\mathscr{A}^{\prime}$ and $\mathscr{A}^{\prime \prime}$ and such that $\partial \mathscr{A}^{\prime}=\partial \mathscr{A}^{\prime \prime}=$ $\mathscr{J}$. The theory also guarantees that one of the components of $\mathbb{R}^{2} \backslash \mathscr{J}$ is bounded 
(the inside) while the other component is unbounded (the outside). Even if from Figure 3 it is clear how to determine the inside and the outside of $\mathscr{J}$, we do not use such an information and we give a proof using only the concept of connectivity, thus avoiding any conclusion based on intuitive arguments.

Let $B\left(P_{2}, \delta\right)$ be the open disc of center $P_{2}$ and radius $\delta$, with

$$
0<\delta<\delta_{0}:=\min \left\{\hat{y}_{2}, \eta^{+}-\eta^{-}, \operatorname{dist}\left(P_{2}, \Gamma_{1}^{\prime}\right)\right\} .
$$

By the choice of $\delta$,

$$
\left.B\left(P_{2}, \delta\right) \cap \mathscr{J}=B\left(P_{2}, \delta\right) \cap \overline{\left[\left(\eta^{+}, 0\right), P_{3}\right]}=\left\{\hat{x}_{2}\right\} \times\right] \hat{y}_{2}-\delta, \hat{y}_{2}+\delta[.
$$

Hence, the two open connected and disjoint sets

$$
\mathscr{B}^{-}:=\left\{(x, y) \in B\left(P_{2}, \delta\right): x<\hat{x}_{2}=\eta^{+}\right\}, \mathscr{B}^{+}:=\left\{(x, y) \in B\left(P_{2}, \delta\right): x>\hat{x}_{2}=\eta^{+}\right\},
$$

such that $\mathscr{B}^{-} \cup \mathscr{B}^{+}=B\left(P_{2}, \delta\right) \backslash \mathscr{J}$, must belong to the two different components of $\mathbb{R}^{2} \backslash \mathscr{J}$ (being $\mathscr{J}$ the common boundary of $\mathscr{A}^{\prime}$ and $\mathscr{A}^{\prime \prime}$ ). Just to fix the ideas, suppose that

$$
\mathscr{B}^{-} \subset \mathscr{A}^{\prime} \text { and } \mathscr{B}^{+} \subset \mathscr{A}^{\prime \prime} \text {. }
$$

Now, we observe that the point $P_{4}$ and $\mathscr{B}^{+}$are in the same component of $\mathbb{R}^{2} \backslash \mathscr{J}$. Indeed, the polygonal arc $\overline{\left[P_{4},\left(\hat{x}_{2}+(\delta / 2), \hat{y}_{4}\right)\right]} \cup \overline{\left[\left(\hat{x}_{2}+(\delta / 2), \hat{y}_{2}\right),\left(\hat{x}_{2}+(\delta / 2), \hat{y}_{4}\right)\right]}$ has empty intersection with $\mathscr{J}$ (recall that all the points of $\Gamma_{1}^{\prime}$ are strictly below the line $\left.y=\hat{y}_{4}=M_{1}\right)$ and connects $P_{4}$ with the point $\left(\hat{x}_{2}+(\delta / 2), \hat{y}_{2}\right) \in \mathscr{B}^{+}$. Hence, $P_{4} \in \mathscr{A}^{\prime \prime}$.

On the other hand, by the continuity of the map $\left[\alpha_{0}, \beta_{0}\right] \mapsto \Phi_{0}^{\tau}(r, 0)$, there exists $\varepsilon>0$ (with $\left.\varepsilon<\beta_{0}-\alpha_{0}\right)$ such that $Q:=\Phi_{0}^{\tau}\left(\beta_{0}-\varepsilon, 0\right) \in \mathscr{B}^{-} \in \mathscr{A}^{\prime}$.

Finally, setting

$$
\Gamma_{0}^{\prime \prime}:=\left\{\Phi_{0}^{\tau}(r, 0): r \in\left[\alpha_{0}, \beta_{0}-\varepsilon\right]\right\} \subset \Gamma_{0}^{\prime},
$$

we have that $\Gamma_{0}^{\prime \prime}$ is a continuum joining $P_{4} \in \mathscr{A}^{\prime \prime}$ with $Q \in \mathscr{A}^{\prime}$. Hence $\mathscr{J} \cap \Gamma_{0}^{\prime \prime} \neq \emptyset$ and this nonempty intersection is exactly $\Gamma_{0}^{\prime \prime} \cap \Gamma_{1}^{\prime}=\Gamma_{0}^{\prime} \cap \Gamma_{1}^{\prime}$. This concludes the proof.

Proof of Theorem 3.2. The proof if precisely the same as that of Theorem 3.1, by using Lemma 4.2 in the variant proposed in Remark 4.1 when $r_{0}>0$ (i.e., the case in which there are solutions presenting a blow-up in the interval $[0, \tau])$. 


\section{Final remarks}

We conclude the paper with a couple of remarks. We always deal with the equation

$$
x^{\prime \prime}=f(x)\left(x^{\prime}\right)^{2}+a(t),
$$

where, as usual, we take $f: \mathbb{R}_{0}^{+} \rightarrow \mathbb{R}$ continuous.

First of all, as already discussed in the Introduction, we recall that our results for the Neumann problem can be used to produce periodic solutions, if we further assume that the weight function $a(t)$ satisfies a symmetry condition. For instance, an explicit corollary of Theorem 3.1 is the following.

Corollary 5.1 Let $f(x)$ satisfy $\left(f_{\infty}\right),\left(F_{\infty}\right)$ and $\left(f_{0}\right)$. Moreover, assume that a $\in$ $L_{\mathrm{loc}}^{1}(\mathbb{R})$ is an even $T$-periodic function (that is, $a(t+T)=a(t)=a(-t)$ for a.e. $t \in \mathbb{R})$ satisfying, for some $\left.\tau^{\prime} \in\right] 0, T / 2[$,

$$
a(t) \geq 0, a(t) \not \equiv 0 \text { on }\left[0, \tau^{\prime}\right] \quad \text { and } \quad a(t) \leq 0, a(t) \not \equiv 0 \text { on }\left[\tau^{\prime}, T / 2\right]
$$

and

$$
\int_{0}^{T} a(t) d t<0
$$

Finally, we suppose that $(A F)$ holds true. Then, equation (5.1) has at least one even $T$-periodic solution, which is decreasing on each interval of the type $[k T-T / 2, k T]$ and increasing on each interval of the type $[k T, k T+T / 2]$, for $k \in \mathbb{Z}$.

Proof. Since $a(t)$ is even and $T$-periodic, one has

$$
\int_{0}^{T / 2} a(t) d t=\frac{1}{2} \int_{0}^{T} a(t) d t<0
$$

Hence, it is possible to apply Theorem 3.1 to produce an increasing solution $[0, T / 2] \ni$ $t \mapsto x(t)$ to (5.1) satisfying the Neumann boundary conditions $x^{\prime}(0)=x^{\prime}(T / 2)=0$. Then, $t \mapsto x(|t| \bmod T)$ is an even $T$-periodic solution of (5.1).

The usual care has to be used in considering assumption $(A F)$. Indeed, if $A(t)=$ 0 for $t \in\left[0, \sigma_{0}\right]$ with $\sigma_{0}<\tau$, one has to solve the Neumann problem on $\left[\sigma_{0}, T / 2\right]$ to produce a $T$-periodic solution which is decreasing on $\left[-T / 2,-\sigma_{0}\right]$, constant on $\left[-\sigma_{0}, \sigma_{0}\right]$ and increasing on $\left[\sigma_{0}, T / 2\right]$.

It is worth noticing that, by a time translation, one can enter in the setting of Corollary 5.1 also when the weight function $a(t)$ is $T$-periodic, satisfies $a(\sigma+t)=$ $a(\sigma-t)$ for some $\sigma \in\left[0, T\left[\right.\right.$ and a.e. $t \in \mathbb{R}$ and, for a suitable $\left.\tau^{\prime} \in\right] 0, T / 2[$,

$$
a(t) \geq 0, a(t) \not \equiv 0 \text { on }\left[\sigma, \sigma+\tau^{\prime}\right] \quad \text { and } \quad a(t) \leq 0, a(t) \not \equiv 0 \text { on }\left[\sigma+\tau^{\prime}, \sigma+T / 2\right] .
$$


In this case, one solves the Neumann boundary value problem on $[\sigma, \sigma+T / 2]$ in order to produce $T$-periodic solutions which are even symmetric with respect to $\sigma$. For instance, a piecewise constant weight function of the form $a(t)=a^{+}>0$ for $t \in[0, \tau]$ and $a(t)=-a^{-}<0$ for $t \in[\tau, T]$ satisfies the previous conditions with $\sigma=\tau^{\prime}=\tau / 2$.

Of course, variants of Corollary 5.1 can be given using Theorem 3.2 instead of Theorem 3.1.

We now briefly touch on the question of the uniqueness/multiplicity of the solutions to the Neumann boundary value problem associated to (5.1). A possible uniqueness result is the following, valid for an arbitrary weight function $a \in L^{1}(0, T)$ (that is, we do not assume any special shape for $a(t)$ ).

Proposition 5.1 Assume that $f(x)$ is of class $C^{1}$ and $f^{\prime}(x)>0$ for every $x>0$. Then, there exists at most one solution to (5.1) satisfying the Neumann boundary conditions $x^{\prime}(0)=x^{\prime}(T)=0$.

Proof. Assume that $x_{1}(t)$ and $x_{2}(t)$ are distinct solutions and set $y(t)=x_{1}(t)-x_{2}(t)$. A simple computation shows that

$$
y^{\prime \prime}-w(t) y^{\prime}=\left(f\left(x_{1}(t)\right)-f\left(x_{2}(t)\right)\right) x_{2}^{\prime}(t)^{2}, \quad \text { for a.e. } t \in[0, T],
$$

with $w(t):=f\left(x_{1}(t)\right)\left(x_{1}^{\prime}(t)+x_{2}^{\prime}(t)\right)$. Just to fix the ideas, suppose that $y\left(t^{*}\right)>0$ for some $t^{*} \in[0, T]$ (if $y\left(t^{*}\right)<0$ the argument is symmetric) and let $\left[t_{1}, t_{2}\right] \subset[0, T]$ be the maximal interval containing $t^{*}$ such that $y(t) \geq 0$ for every $t \in\left[t_{1}, t_{2}\right]$. In view of the maximality of $\left[t_{1}, t_{2}\right]$ and the Neumann boundary conditions on $[0, T]$, it is easy to see that

$$
y^{\prime}\left(t_{2}\right) \leq 0 \leq y^{\prime}\left(t_{1}\right) .
$$

Moreover, since $f^{\prime}(x)>0,(5.2)$ implies that

$$
\frac{d}{d t}\left(y^{\prime} \exp (-W(t))\right)=\exp (-W(t))\left(y^{\prime \prime}-w(t) y^{\prime}\right) \geq 0, \quad \text { for a.e. } t \in\left[t_{1}, t_{2}\right],
$$

where $W(t):=\int_{0}^{t} w(\xi) d \xi$. Integrating, we obtain

$$
y^{\prime}\left(t_{2}\right) \exp \left(-W\left(t_{2}\right)\right) \geq y^{\prime}\left(t_{1}\right) \exp \left(-W\left(t_{1}\right)\right),
$$

so that, combining with (5.3), $y^{\prime}(t)=0$ for every $t \in\left[t_{1}, t_{2}\right]$. Hence $y(t)=y\left(t^{*}\right)>0$ for every $t \in\left[t_{1}, t_{2}\right]$ and $\left[t_{1}, t_{2}\right]=[0, T]$. From (5.2) we thus obtain

$$
0=\left(f\left(x_{2}(t)+y\left(t^{*}\right)\right)-f\left(x_{2}(t)\right)\right) x_{2}^{\prime}(t)^{2}, \quad \text { for every } t \in[0, T] .
$$


This is impossible, since $x_{2}^{\prime}(t) \not \equiv 0$ (otherwise, $\left.a(t) \equiv 0\right)$ and $f(x)$ is strictly increasing.

However, the global condition $f^{\prime}>0$ is in strong contrast with the situation considered in the present paper (compare with $\left(f_{0}\right)$ and $\left(f_{\infty}\right)$ ) so that Proposition 5.1 is not of much help in our context. On the other hand, one can easily see that Proposition 5.1 implies the uniqueness for the positive solution to the Neumann problem associated to $u^{\prime \prime}+a(t) u^{p}=0$, with $0<p<1$, as already shown, also in the PDEs setting, in [4] (using an argument very similar to the one in Proposition 5.1). It is also worth noticing that if $f^{\prime}>0$ on an interval $J^{\prime} \subset \mathbb{R}_{0}^{+}$then the same argument of Proposition 5.1 yields the uniqueness for solutions to the Neumann problem with range in $J^{\prime}$.

We can also observe that the numerical simulation exhibited in Figure 1 seems to suggest that the uniqueness is possible also in cases in which $f^{\prime}$ is not positive (notice that in our example we have indeed $f^{\prime}(x)<0$ for every $x>0$ ). Hence, no more than one solution can be probably expected in Theorem 3.1 (as well as in Theorem 3.2). This could be related to the simple shape of the graph of $a(t)$ : we mention, for instance, that in [12] a uniqueness result is given for (the periodic problem associated to) $u^{\prime \prime}-\frac{a(t)}{u^{3}}=0$ when $a(t)$ is a piecewise constant function satisfying (A2). On the other hand, when the graph of $a(t)$ has a more complex shape, multiple solutions can appear. For instance, in [8] it is proved the existence of three positive solutions to the Neumann problem for $u^{\prime \prime}+a(t) u^{p}=0$, with $p>1$, when $a(t)$ has two positive humps (separated by a negative hump) and large negative mean value.

Acknowledgement The authors thank the referee for the careful reading of the manuscript and for the interesting remarks.

\section{References}

[1] S. Alama and G. Tarantello, On semilinear elliptic equations with indefinite nonlinearities, Calc. Var. Partial Differential Equations 1 (1993), 439-475.

[2] H. Amann and J. López-Gómez, A priori bounds and multiple solutions for superlinear indefinite elliptic problems, J. Differential Equations 146 (1998), 336-374.

[3] C. Bandle, M.A. Pozio and A. Tesei, The asymptotic behavior of the solutions of degenerate parabolic equations, Trans. Amer. Math. Soc. 303 (1987), 487501. 
[4] C. Bandle, M.A. Pozio and A. Tesei, Existence and uniqueness of solutions of nonlinear Neumann problems, Math. Z. 199 (1988), 257-278.

[5] H. Berestycki, I. Capuzzo-Dolcetta and L. Nirenberg, Superlinear indefinite elliptic problems and nonlinear Liouville theorems, Topol. Methods Nonlinear Anal. 4 (1994), 59-78.

[6] H. Berestycki, I. Capuzzo-Dolcetta and L. Nirenberg, Variational methods for indefinite superlinear homogeneous elliptic problems, NoDEA Nonlinear Differential Equations Appl. 2 (1995), 553-572.

[7] D. Bonheure, J.M. Gomes and P. Habets, Multiple positive solutions of superlinear elliptic problems with sign-changing weight, J. Differential Equations 214 (2005), 36-64.

[8] A. Boscaggin, A note on a superlinear indefinite Neumann problem with multiple positive solutions, J. Math. Anal. Appl. 377 (2011), 259-268.

[9] A. Boscaggin and F. Zanolin, Pairs of positive periodic solutions of second order nonlinear equations with indefinite weight, J. Differential Equations 252 (2012), 2900-2921.

[10] A. Boscaggin and F. Zanolin, Positive periodic solutions of second order nonlinear equations with indefinite weight: multiplicity results and complex $d y$ namics, J. Differential Equations 252 (2012), 2922-2950.

[11] A. Boscaggin and F. Zanolin, Pairs of nodal solutions for a class of nonlinear problems with one-sided growth conditions, Adv. Nonlinear Stud. 13 (2013), $13-54$.

[12] J.L. Bravo and P.J. Torres, Periodic solutions of a singular equation with indefinite weight, Adv. Nonlinear Stud. 10 (2010), 927-938.

[13] G.J. Butler, Rapid oscillation, nonextendability, and the existence of periodic solutions to second order nonlinear ordinary differential equations, J. Differential Equations 22 (1976), 467-477.

[14] C. Chicone, The monotonicity of the period function for planar Hamiltonian vector fields, J. Differential Equations 69 (1987), 310-321.

[15] J. Chu, P.J. Torres and M. Zhang, Periodic solutions of second order non-autonomous singular dynamical systems, J. Differential Equations 239 (2007), 196-212. 
[16] M. Gaudenzi, P. Habets and F. Zanolin, Positive solutions of singular boundary value problems with indefinite weight, Bull. Belg. Math. Soc. Simon Stevin 9 (2002), 607-619.

[17] M. Gaudenzi, P. Habets and F. Zanolin, An example of a superlinear problem with multiple positive solutions, Atti Sem. Mat. Fis. Univ. Modena 51 (2003), 259-272.

[18] M. Gaudenzi, P. Habets and F. Zanolin, Positive solutions of superlinear boundary value problems with singular indefinite weight, Commun. Pure Appl. Anal. 2 (2003), 411-423.

[19] M. Gaudenzi, P. Habets and F. Zanolin, A seven-positive-solutions theorem for a superlinear problem, Adv. Nonlinear Stud. 4 (2004), 149-164.

[20] P.M. Girão and J.M. Gomes, Multibump nodal solutions for an indefinite superlinear elliptic problem, J. Differential Equations 247 (2009), 1001-1012.

[21] R. Gómez-Reñasco and J. López-Gómez, The effect of varying coefficients on the dynamics of a class of superlinear indefinite reaction-diffusion equations, J. Differential Equations 167 (2000), 36-72.

[22] W.B. Gordon, Conservative dynamical systems involving strong forces, Trans. Amer. Math. Soc. 204 (1975), 113-135.

[23] J.K. Hale, Ordinary Differential Equations. Second edition, Robert E. Krieger Publishing Co., Inc., Huntington, N.Y., 1980.

[24] M. Henle, A Combinatorial Introduction to Topology, Dover Publications, Inc., New York, 1994.

[25] E. Kamke, Differentialgleichungen: Lösungsmethoden und Lösungen, Stuttgart, Teubner, 1977.

[26] K. Lan and J.R.L. Webb, Positive solutions of semilinear differential equations with singularities, J. Differential Equations 148 (1998), 407-421.

[27] V.K. Le and K. Schmitt, Minimization problems for noncoercive functionals subject to constraints, Trans. Amer. Math. Soc. 347 (1995), 4485-4513.

[28] J. López-Gómez, Varying bifurcation diagrams of positive solutions for a class of indefinite superlinear boundary value problems, Trans. Amer. Math. Soc. 352 (2000), 1825-1858. 
[29] J.S. Muldowney and D. Willett, An elementary proof of the existence of solutions to second order nonlinear boundary value problems, SIAM J. Math. Anal. 5 (1974), 701-707.

[30] D. Papini and F. Zanolin, A topological approach to superlinear indefinite boundary value problems, Topol. Methods Nonlinear Anal. 15 (2000), 203233.

[31] A. Pascoletti and F. Zanolin, A path crossing lemma and applications to nonlinear second order equations under slowly varying perturbations, Le Matematiche (Catania) 65 (2010), 121-168.

[32] L.A. Peletier and A. Tesei, Global bifurcation and attractivity of stationary solutions of a degenerate diffusion equation, Adv. in Appl. Math. 7 (1986), 435-454.

[33] M. Sabatini, On the period function of $x^{\prime \prime}+f(x) x^{\prime 2}+g(x)=0$, J. Differential Equation 196 (2004), 151-168.

[34] S. Terracini and G. Verzini, Oscillating solutions to second-order ODEs with indefinite superlinear nonlinearities, Nonlinearity 13 (2000), 1501-1514.

[35] A. J. Ureña, Periodic solutions of singular equations with indefinite weight, communication delivered at the conference "Qualitative Theory of Nonlinear Differential Equations 2013”, Trieste. 